Pacific

Journal of

Mathematics

FULL EXTENSIONS AND APPROXIMATE UNITARY EQUIVALENCE

HUAXIN LIN

Volume $229 \quad$ No. 2

February 2007 


\title{
FULL EXTENSIONS AND APPROXIMATE UNITARY EQUIVALENCE
}

\author{
HUAXIN LIN
}

\begin{abstract}
Let $A$ be a unital separable amenable $C^{*}$-algebra and let $C$ be a unital $C^{*}$ algebra with a certain infinite property. We show that two full monomorphisms $h_{1}, h_{2}: A \rightarrow C$ are approximately unitarily equivalent if and only if $\left[h_{1}\right]=\left[h_{2}\right]$ in $K L(A, C)$. Let $B$ be a nonunital but $\sigma$-unital $C^{*}$-algebra for which $M(B) / B$ has a certain infinite property. We prove that two full essential extensions are approximately unitarily equivalent if and only if they induce the same element in $K L(A, M(B) / B)$. The set of approximately unitarily equivalence classes of full essential extensions forms a group. If $\boldsymbol{A}$ satisfies the Universal Coefficient Theorem, the group can be identified with $K L(A, M(B) / B)$.
\end{abstract}

\section{Introduction}

The study of $C^{*}$-algebra extensions originated in the study of essentially normal operators on the infinite-dimensional separable Hilbert space. The original BrownDouglas-Fillmore theory gives a classification of essential normal operators via certain Fredholm related indices (see [Brown et al. 1973b]). Later the theory was expanded to yield a classification of essential extensions of $C(X)$ by compact operators [Brown et al. 1973a; Brown 1984]. The study of $C^{*}$-algebra extensions developed into Kasparov's $K K$-theory and its applications can be found not only in operator theory and operator algebras but also in differential geometry and noncommutative geometry.

Let

$$
0 \rightarrow B \rightarrow E \rightarrow A \rightarrow 0
$$

be an essential extension of $A$ by $B$. The extension is determined by a monomorphism $\tau: A \rightarrow M(B) / B$, the Busby invariant. When $B$ is a $\sigma$-unital stable $C^{*}$ algebra, $K K^{1}(A, B)$ gives a complete classification of such essential extensions, up to stable unitary equivalence. However, $K K^{1}(A, B)$ gives little information, if any, about unitary equivalence classes of these mentioned extensions when $B \neq \mathscr{K}$ in

MSC2000: 46L05, 46L35.

Keywords: extension of $C^{*}$-algebras, simple $C^{*}$-algebras.

Research partially supported by NSF grants DMS 0097903 and DMS 00355273. 
general. There are examples in which $K K^{1}(A, B)=\{0\}$ but inequivalent nontrivial extensions exist [Lin 1998, Example 0.6]. There are also examples in which there are infinitely many inequivalent classes of trivial extensions [Lin 1995b, 7.4 and 7.5]. When $B$ is not stable, $K K^{1}(A, B)$ certainly should not be used to understand unitary equivalence classes of the essential extensions mentioned above.

There are a number of results in classification of essential extensions (up to unitary equivalence or approximate unitary equivalence) when $B \neq \mathscr{K}$. Kirchberg's results [1996] on extensions in which $B$ is a nonunital purely infinite simple $C^{*}$ algebra show that $K K^{1}(A, B)$ can be used to compute unitary equivalence classes of those extensions. If $B$ is a nonunital but $\sigma$-unital simple $C^{*}$-algebra with continuous scale (see item (6) on page 391), then $M(B) / B$ is simple. A classification of essential extensions of a separable amenable $C^{*}$-algebra $A$ by $B$ (up to approximate unitary equivalence) was obtained in [Lin 2005b] (for some special cases in which $A=C(X)$, a classification up to unitary equivalence was obtained in [Lin 1995a; $1995 b ; 1997]$. In this case, $B$ may not be stable, so $K K^{1}(A, B)$ is not used as an invariant for essential extensions. Results about extensions of AF-algebras can be found in [Brown and Elliott 1982; Goodearl and Handelman 1982; Elliott and Handelman 1989].

Here we study full essential extensions. These are essential extensions $\tau: A \rightarrow$ $M(B) / B$ such that $\tau(a)$ is a full element for each nonzero element $a \in A$. Since the Calkin algebra $M(\mathscr{K}) / \mathscr{K}$ is simple, all essential extensions by $\mathscr{K}$ are full. If $B$ is a nonunital but $\sigma$-unital purely infinite simple $C^{*}$-algebra, $M(B) / B$ is also simple. Therefore essential extensions by those $C^{*}$-algebras are all full. The homogeneous extensions of $A$ by $C(X) \otimes \mathscr{K}$ studied by Pimsner, Popa and Voiculescu [Pimsner et al. 1979; 1980] are all full extensions. In these three cases, $B$ is stable. There are nonstable, nonunital but $\sigma$-unital $C^{*}$-algebras that have continuous scale. In that case essential extensions by these $C^{*}$-algebras are also full. Furthermore, if $A$ is a unital simple $C^{*}$-algebra and if the monomorphism $\tau: A \rightarrow M(B) / B$ is unital, then the essential extension induced by $\tau$ is always full for any nonunital $C^{*}$-algebra $B$.

With a technical condition on $M(B) / B$, we show that two full essential extensions are approximately unitarily equivalent if they induce the same element in $K L(A, M(B) / B)$ (see Theorem 2.5), provided that $A$ is amenable and separable. When $A$ is assumed to satisfy the (Approximate) Universal Coefficient Theorem, we show that there is a bijective correspondence between approximate unitary equivalence classes of essential and full extensions and $K L(A, M(B) / B)$. The advantage of studying these full extensions is that full extensions (in these cases) are "approximately absorbing". For stable $B$, we show that $K K^{1}(A, B)$ classifies the unitary equivalence classes of full essential extensions. In this case, full extensions are "purely large" in the sense of [Elliott and Kucerovsky 2001]. 
Section 2 describes the main results in this paper and introduces the technical conditions $\mathrm{P} 1, \mathrm{P} 2$ and $\mathrm{P} 3$. Section 3 shows that the corona algebras $M(B) / B$ of many stable $C^{*}$-algebras satisfy these conditions. In Section 4 , we show that there are examples of nonstable, nonunital and $\sigma$-unital $C^{*}$-algebras $B$ for which $M(B) / B$ has properties $\mathrm{P} 1, \mathrm{P} 2$ and $\mathrm{P} 3$. In Section 5 we give a few modified versions of some known results concerning amenable contractive completely positive linear maps. Section 6 discusses certain commutants in the ultrapower of corona algebras. In Section 7 we prove Theorem 2.5, mentioned above. In Section 8 we prove other main results described in Section 2.

Conventions and definitions. (1) Ideals are always closed and two-sided.

(2) Let $A$ be a $C^{*}$-algebra and let $p, q \in A$ be two projections. We write $p \sim q$ if there exists $v \in A$ such that $v^{*} v=p$ and $v v^{*}=q$.

(3) Let $A$ and $B$ be $C^{*}$-algebras and let $L_{1}, L_{2}: A \rightarrow B$ be linear maps. Take $\mathscr{F} \subset A$ and $\varepsilon>0$. We write $L_{1} \sim_{\varepsilon} L_{2}$ on $\mathscr{F}$ if

$$
\left\|L_{1}(a)-L_{2}(a)\right\|<\varepsilon \quad \text { for all } a \in \mathscr{F} \text {. }
$$

(4) Let $A$ and $B$ be $C^{*}$-algebras. A contractive completely positive linear map $L: A \rightarrow B$ is said to amenable if for $\varepsilon>0$ and any finite subset $\mathscr{F} \subset A$, there exists an integer $n>0$ and two contractive completely positive linear maps $\phi: A \rightarrow M_{n}$ and $\psi: M_{n} \rightarrow A$ such that

$$
\psi \circ \phi \sim_{\varepsilon} L \quad \text { on } \mathscr{F} .
$$

(5) A $C^{*}$-algebra $A$ is said to be amenable (or nuclear) if $\mathrm{id}_{A}$ is amenable.

(6) Let $B$ be a nonunital but $\sigma$-unital simple $C^{*}$-algebra. $B$ is said to have continuous scale if there exists an approximate identity $\left\{e_{n}\right\}$ of $B$ with $e_{n+1} e_{n}=e_{n}$ such that, for each nonzero element $b \in B$, there exists an integer $n>0$ for which $e_{n+m}-e_{n} \lesssim b$ for all $m$; see [Lin 2004b].

Let $e \in B$ be a nonzero projection and $T_{e}(B)$ the set of all traces $t$ on $B$ for which $t(e)=1$. Let $B$ be a separable nonunital simple $C^{*}$-algebra with real rank 0 , stable rank 1 and weakly unperforatated $K_{0}(B)$. If $\sup _{n}\left\{t\left(e_{n}\right)\right\}$ is a continuous function on $T_{e}(B)$, then $B$ has continuous scale.

(7) Let $\left\{A_{n}\right\}$ be a sequence of $C^{*}$-algebras. Denote by $c_{0}\left(\left\{A_{n}\right\}\right)$ the $C^{*}$ direct sum of $\left\{A_{n}\right\}$ and by $l^{\infty}\left(\left\{A_{n}\right\}\right)$ the $C^{*}$ - product of $\left\{A_{n}\right\}$. Let $q_{\infty}\left(\left\{A_{n}\right\}\right)$ be the quotient

$$
q_{\infty}\left(\left\{A_{n}\right\}\right)=l^{\infty}\left(\left\{A_{n}\right\}\right) / c_{0}\left(\left\{A_{n}\right\}\right) .
$$

When $A=A_{n}$ for all $n$, we write $c_{0}(A), l^{\infty}(A)$ and $q_{\infty}(A)$ for simplicity. 
(8) For each integer $n>0$, define $f_{n} \in C_{0}((0, \infty))$ by

$$
f_{n}(t)= \begin{cases}1 & \text { if } t \geq 1 / n \\ \text { linear } & \text { if } 1 /(n+1) \leq t<1 / n \\ 0 & \text { if } 0 \leq t<1 /(n+1)\end{cases}
$$

(9) An element $a$ in a $C^{*}$-algebra $A$ is said be full if the ideal generated by $a$ is $A$ itself. Let $A$ and $B$ be $C^{*}$-algebras and let $h: A \rightarrow B$ be a monomorphism. The monomorphism $h$ is said to be full if $h(a)$ is full for every nonzero $a \in A$.

(10) Let $a \in A_{+}$be a nonzero element, we write $\operatorname{Her}(a)$ for the hereditary $C^{*}$ subalgebra $\overline{a A a}$ generated by $a$.

\section{Main results}

Property P1. Let $B$ be a unital $C^{*}$-algebra. We say that $B$ has property P1 if for every full element $b \in B$ there exist $x, y \in B$ such that $x b y=1$. If $b$ is positive, it is easy to see that $x b y=1$ implies that there is $z \in B$ such that $z^{*} b z=1$.

It is obvious that an element $b$ is full if and only if $b^{*} b$ is full. It follows that $B$ has property $\mathrm{P} 1$ if and only if for every full element $0 \leq b \leq 1$, there exists $x \in B$ such that $x^{*} b x=1$.

Every unital purely infinite simple $C^{*}$-algebra has property $\mathrm{P} 1$.

Many other unital $C^{*}$-algebras have property P1. Let $A$ be a unital $C^{*}$-algebra and $B=A \otimes \mathscr{K}$. In the next section we will show that $M(B)$ and $M(B) / B$ have property $\mathrm{P} 1$ for many such $B$. In Section 3 we will show that, for some nonstable (but $\sigma$-unital) $C^{*}$-algebra $C, M(C)$ and $M(C) / C$ can also have property $\mathrm{P} 1$.

Property P2. Let $B$ be a unital $C^{*}$-algebra. We say that $B$ has property P2 if 1 is proper infinite, that is, if there is a projection $p \neq 1$ and partial isometries $w_{1}, w_{2} \in B$ such that $w_{1}^{*} w_{1}=1, w_{1} w_{1}^{*}=p, w_{2}^{*} w_{2}=1$ and $w_{2} w_{2}^{*} \leq 1-p$.

In this case it is easy to see that, for each integer $n \geq 2$, there are mutually orthogonal and mutually equivalent projections $s_{11}, s_{22}, \ldots, s_{n n}$ such that $1_{B} \geq$ $\sum_{i=1}^{n} s_{i i}$ and there exists an isometry $Z \in B$ such that $Z^{*} Z=1_{B}$ and $Z Z^{*}=s_{11}$. Let $C=s_{11} B s_{11}$. Then we may write $M_{n}(C) \subset B$.

It is clear that if $B$ is stable then $M(B)$ and $M(B) / B$ have property $\mathrm{P} 2$.

Proposition 2.1. Let $B$ be a unital $C^{*}$-algebra having property $P 1$. If $B$ contains two mutually orthogonal full elements, $B$ has property $P 2$.

Proof. Let $0 \leq a, b \leq 1$ be two mutually orthogonal full elements in $B$. Since $B$ has property $\mathrm{P} 1$, there are $x, y \in B$ such that $x^{*} a x=1$ and $y^{*} b y=1$. Set $v_{1}=a^{1 / 2} x$ and $v_{2}=b^{1 / 2} y$. Then $v_{i}^{*} v_{i}=1$ and $s_{11}=v_{1} v_{1}^{*}$ and $s_{22}=v_{2} v_{2}^{*}$ are two projections. Thus $B$ has property $\mathrm{P} 2$. 
Every purely infinite $C^{*}$-algebra (not necessary simple; see [Kirchberg and Rørdam 2000]) has properties P1 and P2.

Property P3. Let $B$ be a unital $C^{*}$-algebra. We say that $B$ has property P3 if, for any separable $C^{*}$-subalgebra $A \subset B$, there exists a sequence of sequences of elements $\left\{\left\{a_{n}^{(i)}\right\}: i=1,2, \ldots\right\}$ in $B$ satisfying these properties:

(a) $0 \leq a_{n}^{(i)} \leq 1$ for all $i$ and $n$;

(b) $\lim _{n \rightarrow \infty}\left\|a_{n}^{(i)} c-c a_{n}^{(i)}\right\|=0$ for all $i$ and all $c \in A$;

(c) $\lim _{n \rightarrow \infty}\left\|a_{n}^{(i)} a_{n}^{(j)}\right\|=0$ if $i \neq j$;

(d) $\left\{a_{n}^{(i)}\right\}$ is a full element in $l^{\infty}(B)$, for all $i$.

Even though property P3 looks more complicated than P1 and P2, it will be shown in Proposition 3.13 that $M(B) / B$ has property $\mathrm{P} 3$ for all $B=C \otimes \mathscr{K}$, where $C$ is a unital $C^{*}$-algebra, for all $B$ having continuous scale, and for many other nonunital $\sigma$-unital $C^{*}$-algebras $B$.

Proposition 2.2. Let $B=C \otimes C_{1}$, where $C_{1}$ is a unital separable amenable purely infinite simple $C^{*}$-algebra. Then $B$ has properties $P 1, P 2$ and $P 3$.

Let $B$ be a nonunital but $\sigma$-unital $C^{*}$-algebra and let $A$ be a unital separable amenable $C^{*}$-algebra. We study essential extensions of the following form:

$$
0 \rightarrow B \rightarrow E \rightarrow A \rightarrow 0 .
$$

Using the Busby invariant, we study monomorphisms $\tau: A \rightarrow M(B) / B$. We will only consider the case in which the corona algebra $M(B) / B$ has properties $\mathrm{P} 1, \mathrm{P} 2$ and P3.

Definition 2.3. An essential extension $\tau: A \rightarrow M(B) / B$ is said to be full if $\tau$ is a full monomorphism. An extension $\tau$ is weakly unital if $\tau$ is unital monomorphism. If $A$ is a unital simple $C^{*}$-algebra then every weakly unital essential extension is full. If $M(B) / B$ is simple, every essential extension is full.

Definition 2.4. Let $A$ be a unital separable $C^{*}$-algebra and $C$ a unital $C^{*}$-algebra. Let $h_{1}, h_{2}: A \rightarrow C$ be homomorphisms. We say $h_{1}$ and $h_{2}$ are approximately unitarily equivalent if there exists a sequence of partial isometries $u_{n} \in C$ such that $u_{n}^{*} h_{1}\left(1_{A}\right) u_{n}=h_{2}\left(1_{A}\right), u_{n} h_{2}\left(1_{A}\right) u_{n}^{*}=h_{1}\left(1_{A}\right)$ and

$$
\lim _{n \rightarrow \infty}\left\|\operatorname{ad} u_{n} \circ h_{1}(a)-h_{2}(a)\right\|=0 \quad \text { for all } a \in A \text {. }
$$

Note that if both $h_{1}$ and $h_{2}$ are unital, the $u_{n}$ can be chosen to be unitaries.

Let $B$ be a nonunital but $\sigma$-unital $C^{*}$-algebra. Two essential extensions of $A$ by $B$ are said to be approximately unitarily equivalent if the corresponding Busby invariants $\tau_{1}, \tau_{2}: A \rightarrow M(B) / B$ are approximately unitarily equivalent. 
Recall that $\tau: A \rightarrow M(B) / B$ is trivial if there is a monomorphism $h: A \rightarrow M(B)$ such that $\pi \circ h=\tau$, where $\pi: M(B) \rightarrow M(B) / B$ is the quotient map. In the case that $B=C \otimes \mathscr{K}$, where $C$ is a $\sigma$-unital $C^{*}$-algebra, the invariants $\tau_{1}$ and $\tau_{2}$ are stably unitarily equivalent if there exists a trivial extension $\tau_{0}: A \rightarrow M(B) / B$ and a unitary $u \in M_{2}(M(B) / B)$ such that ad $u \circ\left(\tau_{1} \oplus \tau_{0}\right)=\tau_{2} \oplus \tau_{0}$.

Let $\operatorname{Ext}(A, B)$ be the set of stable unitary equivalence classes of extensions of the form (2-1). When $A$ is a separable amenable $C^{*}$-algebra, $\operatorname{Ext}(A, B)$ can be identified with $K K^{1}(A, B)$. When $A$ satisfies the Universal Coefficient Theorem, $K K^{1}(A, B)$ is computable. However, as mentioned in the introduction, $K K^{1}(A, B)$ may not provide any useful information about unitary equivalence of extensions in general. In particular, when $B$ is not stable, $K K^{1}(A, B)$ should not be used to describe unitary equivalence classes of essential extensions.

The first main result of this paper is the following:

Theorem 2.5. Let $A$ be a unital separable amenable $C^{*}$-algebra and let $B$ be a nonunital but $\sigma$-unital $C^{*}$-algebra such that $M(B) / B$ has properties $P 1, P 2$ and P3. Two full monomorphisms $\tau_{1}, \tau_{2}: A \rightarrow M(B) / B$ are approximately unitarily equivalent if and only if

$$
\left[\tau_{1}\right]=\left[\tau_{2}\right] \quad \text { in } K L(A, M(B) / B) .
$$

We will describe $K L(A, C)$ in Definition 7.1. Theorem 2.5 is an easy corollary of the next theorem.

Theorem 2.6. Let $A$ be a unital separable amenable $C^{*}$-algebra and let $B$ be a unital $C^{*}$-algebra having properties $P 1, P 2$ and $P 3$. Two full monomorphisms $h_{1}, h_{2}: A \rightarrow B$ are approximately unitarily equivalent, i.e., there exists a sequence of partial isometries $u_{n} \in B$ such that $u_{n}^{*} u_{n}=h_{1}\left(1_{A}\right), u_{n} u_{n}^{*}=h_{2}\left(1_{A}\right)$ and

$$
\lim _{n \rightarrow \infty} \operatorname{ad} u_{n} \circ h_{1}(a)=h_{2}(a) \quad \text { for all } a \in A,
$$

if and only if $\left[h_{1}\right]=\left[h_{2}\right]$ in $K L(A, B)$.

Corollary 2.7. Let $A$ be a unital separable amenable simple $C^{*}$-algebra and $B$ a nonunital but $\sigma$-unital $C^{*}$-algebra such that $M(B) / B$ has properties $P 1, P 2$ and $P 3$. Suppose that $\tau_{1}, \tau_{2}: A \rightarrow M(B) / B$ are two weakly unital essential extensions. Then $\tau_{1}$ and $\tau_{2}$ are approximately unitarily equivalent if and only if

$$
\left[\tau_{1}\right]=\left[\tau_{2}\right] \text { in } \operatorname{KL}(A, M(B) / B) .
$$

Definition 2.8. Let $A$ be a unital separable amenable $C^{*}$-algebra and $B$ a unital $C^{*}$-algebra having property $\mathrm{P} 2$. Fix a full monomorphism $j_{o}: A \rightarrow \mathrm{O}_{2} \rightarrow B$. (Note that $\mathrm{P} 2$ implies such full monomorphisms do exist.) Let $h_{1}, h_{2}: A \rightarrow B \otimes \mathscr{K}$ be homomorphisms. We write $h_{1} \sim h_{2}$ if $h_{1} \oplus j_{o}$ is approximately unitarily equivalent 
to $h_{2} \oplus j_{o}$. Denote by $H(A, B)$ the set of $\sim$-equivalence classes of homomorphisms $A \rightarrow B \otimes \mathcal{K}$.

Proposition 2.9. Let $A$ be a unital separable amenable $C^{*}$-algebra and $B$ a unital $C^{*}$-algebra having property $P 2$. Then $H(A, B)$ is a group with zero element $\left[j_{o}\right]$.

Corollary 2.10. Let $A$ be a unital separable amenable $C^{*}$-algebra and $B$ a unital $C^{*}$-algebra having properties $P 1, P 2$ and $P 3$. Denote by $H_{f}(A, B)$ the set of approximate unitary equivalence classes of full monomorphisms from $A$ to $B \otimes \mathcal{K}$. Then $H_{f}(A, B)$ is a group with zero element $\left[j_{o}\right]$.

Definition 2.11. Let $A$ be a unital separable amenable $C^{*}$-algebra and $B$ a nonunital but $\sigma$-unital $C^{*}$-algebra. Denote by $\operatorname{Ext}_{a p}^{f}(A, B)$ the approximate unitary equivalence classes of full essential extensions. Denote by $\tau_{o}: A \rightarrow M(B) / B$ an essential extension that factors through $\mathrm{O}_{2}$. Note that $\left[\tau_{o}\right]=0$ in $K L(A, M(B) / B)$. Suppose that $M(B) / B$ has properties $\mathrm{P} 1, \mathrm{P} 2$ and $\mathrm{P} 3$. By Corollary $2.7, \tau_{o}$ is unique up to approximately unitary equivalence, Let $\tau_{1}, \tau_{2}: A \rightarrow M(B) / B$ be full essential extensions. Since $M(B) / B$ has property $\mathrm{P} 2$, there are partial isometries $z_{1}, z_{2} \in M(B) / B$ such that $z_{1}^{*} z_{1}=1_{M(B) / B}, z_{1} z_{1}^{*}=\tau_{1}\left(1_{A}\right), z_{2}^{*} z_{2}=1_{M(B) / B}$ and $z_{2} z_{2}^{*}=\tau_{2}\left(1_{A}\right)$. Define $\left[\tau_{1}\right]+\left[\tau_{2}\right]=\left[\operatorname{ad} z_{1} \circ \tau_{1} \oplus \operatorname{ad} z_{2} \circ \tau_{2}\right]$.

Note this is well defined, since $\left[\tau_{o}\right]=0$ in $K L(A \cdot M(B) / B)$ and $\operatorname{ad} z_{1} \circ \tau \oplus$ $\operatorname{ad} z_{2} \circ \tau_{o}$ is approximately unitarily equivalent to $\tau$ by Corollary 2.7. With this addition $\mathbf{E x t}_{a p}^{f}(A, B)$ forms a semigroup.

By Corollary 2.10, we have:

Corollary 2.12. Let $A$ be a unital separable amenable $C^{*}$-algebra and $B$ a nonunital but $\sigma$-unital $C^{*}$-algebra for which $M(B) / B$ has properties $P 1, P 2$ and $P 3$. Then $\mathbf{E x t}_{a p}^{f}(A, B)$ is a group with zero element $\left[\tau_{o}\right]$, where $\tau_{o}: A \rightarrow M(B) / B$ is a full monomorphism that factors through $\mathrm{O}_{2}$.

If, furthermore, $A$ satisfies the so-called Approximate Universal Coefficient Theorem (see Definition 7.1 below), we can say more:

Theorem 2.13. Let $A$ be a unital separable amenable $C^{*}$-algebra satisfying the Approximate Universal Coefficient Theorem and let $B$ be a nonunital but $\sigma$-unital $C^{*}$-algebra such that $M(B) / B$ has properties $P 1, P 2$ and $P 3$. Then there is a bijection $\Gamma$ from $\mathbf{E x t}_{a p}^{f}(A, B)$ onto $K L(A, M(B) / B)$.

The Approximate Universal Coefficient Theorem will be briefly reviewed in Definitions Definition 7.1 and Definition 8.1. Note that, when $B$ is not stable, $K_{i}(M(B) / B)$ is very different from $K_{i}(S B)$ [Lin 2005b, 1.7].

In the special case that $B=C \otimes \mathscr{K}$, where $C$ is a unital $C^{*}$-algebra, we have:

Theorem 2.14. Let $A$ be a unital separable amenable $C^{*}$-algebra and set $B=$ $C \otimes \mathscr{K}$, where $C$ is a unital $C^{*}$-algebra such that $M(B) / B$ has the property $P 1$. 
Two full essential extensions $\tau_{1}, \tau_{2}: A \rightarrow M(B) / B$ are unitarily equivalent if and only if

$$
\left[\tau_{1}\right]=\left[\tau_{2}\right] \quad \text { in } K K^{1}(A, B) .
$$

If $x \in K K^{1}(A, B)$, there is a full essential extension $\tau: A \rightarrow M(B) / B$ such that $[\tau]=x$.

Theorem 2.15. Let $A$ be a unital separable amenable $C^{*}$-algebra and set $B=$ $C \otimes \mathcal{K}$, where $C$ is a unital $C^{*}$-algebra for which the tracial state space $T(C)$ is nonempty. Suppose that there is $d>0$ for which $C$ satisfies the following:

(1) If $p, q \in B$ are two projections, the condition $t(p)>d+t(q)$ for all $t \in T(C)$ implies $q \sim p$ in $B$.

(2) If $b \in M_{k}(C)$ is such that $1 \geq b \geq 0$ and $\tau(b)>\alpha+d$ for all $\tau \in T(A)$, there is a projection $e \in \overline{b M_{k}(A) b}$ such that $\tau(e)>\alpha$ for all $\tau \in T(A)$.

Then two full essential extensions $\tau_{1}, \tau_{2}: A \rightarrow M(B) / B$ are unitarily equivalent if and only if

$$
\left[\tau_{1}\right]=\left[\tau_{2}\right]
$$

Remark 2.16. In the case that $B=\mathscr{K}$, Theorem 2.14 is the classical BrownDouglas-Fillmore theorem, and $M(\mathscr{K}) / \mathscr{K}$ is a purely infinite simple $C^{*}$-algebra. It has property P1 (as well as P2 and P3) and every essential extension is full. Let $X$ be a compact metric space with finite dimension $d$. When $B=C(X) \otimes \mathscr{K}, M(B) / B$ has property P1 (Corollary 3.9). Theorems 2.14 and 2.15 deal with the extensions studied by Pimsner, Popa and Voiculescu [Pimsner et al. 1979; 1980]. The case where $B$ is a nonunital purely infinite simple $C^{*}$-algebra was proved by Kirchberg.

Theorem 2.14 is closely related to a result of Elliott and Kucerovsky [2001]; see Remark 8.7 for discussion.

\section{3. $C^{*}$-algebras have properties $\mathrm{P} 1, \mathrm{P} 2$ and $\mathrm{P} 3$}

Let $A$ be a unital $C^{*}$-algebra. Denote by $T(A)$ (or $T$ if no confusion exits) the set of tracial states on $A$. If $t \in T(A)$, we extend $t$ to a trace $(t \otimes T r)$ on $A \otimes M_{n}$ by defining $t\left(\left(a_{i j}\right)=\sum_{i=1}^{n} t\left(a_{i i}\right)\right.$. We further use $t$ for the trace defined on a dense set of $A \otimes \mathscr{K}$. If $a \in A \otimes \mathscr{K}_{+}$, then $t(a)$ is well defined (although it could be infinity). Suppose that $h_{n} \in A \otimes \mathscr{K}_{+}$is such that $h_{n} \nearrow h \in A^{* *}$. Then $t(h)=\lim _{n \rightarrow \infty} t\left(h_{n}\right)$. These conventions will be used in this section.

Lemma 3.1. Let $A$ be a unital $C^{*}$-algebra and I a $\sigma$-unital ideal of $A$. If $a \in$ $(A / I)_{+}$is a full element, there exists a full element $b \in A_{+}$such that $\pi(b)=a$, where $\pi: A \rightarrow A / I$ is the quotient map. 
Proof. This result is certainly known, but we prove it for completeness. Since $a \in(A / I)_{+}$is full, there are $x_{1}, x_{2}, \ldots, x_{m} \in A / I$ such that

$$
\sum_{i=1}^{m} x_{i}^{*} a x_{i}=1 \text {. }
$$

Hence there are $c \in A_{+}$and $y_{1}, y_{2}, \ldots, y_{m} \in A$ such that $\pi(c)=a$ and $1-$ $\sum_{i=1}^{m} y_{i}^{*} c y_{i} \in I$. Let $e$ be a strictly positive element of $I$. Put $b=c+e$. Denote by $J$ the ideal generated by $b$. Since $b \geq c$ and $b \geq e$, both $c$ and $e$ are in $J$. It follows that $I \subset J$. Since $\sum_{i=1}^{m} y_{i}^{*} c y_{i} \in J$, it follows that $1 \in J$. Thus $J=A$, and $b$ is full.

Corollary 3.2. Let $A$ be a unital $C^{*}$-algebra and I a $\sigma$-unital ideal of $A$. If $A$ has property $P 1$, then so does $A / I$.

Lemma 3.3. Let $A$ be a unital $C^{*}$-algebra and let $B=A \otimes \mathscr{K}$. Suppose that $a \in M(B)$ is an element for which $b=\pi(a)$ is full in $M(B) / B$, where $\pi: M(B) \rightarrow$ $M(B) / B$ is the quotient map. Then a is full in $M(B)$. If $M(B) / B$ has property $P 1$, so does $M(B)$.

Proof. There are $x_{1}, x_{2}, \ldots, x_{m} y_{1}, \ldots, y_{m} \in M(B) / B$ such that $\sum_{i=1}^{m} x_{i} b y_{i}=1$. Then there are $w_{1}, w_{2}, \ldots, w_{m}, z_{1}, z_{2}, \ldots, z_{m} \in M(B)$ such that

$$
1-\sum_{i=1}^{m} w_{i} a z_{i} \in B
$$

Let $\left\{e_{i j}\right\}$ be a system of matrix units for $\mathscr{K}$. Put $E_{n}=\sum_{i=1}^{n} e_{i i}$. Then $\left\{E_{n}\right\}$ is an approximate identity consisting of projections. It follows that there exists $n>0$ such that

$$
\left\|\sum_{i=1}^{m}\left(1-E_{n}\right) w_{i} a z_{i}\left(1-E_{n}\right)-\left(1-E_{n}\right)\right\|<\frac{1}{2} .
$$

Thus there exists $s \in\left(1-E_{n}\right) M(B)\left(1-E_{n}\right)$ such that

$$
\sum_{i=1}^{m} s^{*}\left(1-E_{n}\right) w_{i} a z_{i}\left(1-E_{n}\right) s=1-E_{n} .
$$

But there exists $V \in M(B)$ such that $V^{*}\left(1-E_{n}\right) V=1$. Therefore $a$ is full.

For the last statement, we take $m=1$ in the argument above.

Proposition 3.4. Let $B$ be a unital purely infinite simple $C^{*}$-algebra. Then $M(B \otimes$ $\mathscr{K})$ and $M(B \otimes \mathscr{K}) / A \otimes \mathscr{K}$ have property $P 1$.

Proof. It follows from [Zhang 1992] that $M(B \otimes \mathscr{K}) /(B \otimes \mathscr{K})$ is purely infinite and simple. Therefore $M(B \otimes \mathscr{K}) / B \otimes \mathscr{K}$ has property P1. Now Lemma 3.3 implies that $M(B \otimes \mathscr{K})$ has property $\mathrm{P} 1$. 
Theorem 3.5. Let $B=A \otimes \mathscr{K}$, where $A$ is a unital separable $C^{*}$-algebra for which $T(A) \neq \varnothing$. Let $d>0$. Suppose A satisfies the following:

(1) If $p, q \in B$ are two projections then $t(p)>d+t(q)$ for all $t \in T(A)$ implies $p \precsim q$ in $B$.

(2) If $1 \geq b \geq 0$ in $M_{k}(A)$ such that $\tau(b)>\alpha+d$ for all $\tau \in T(A)$ (and some $\alpha>0)$, then there is a projection $e \in \overline{b M_{k}(A) b}$ such that $\tau(e)>\alpha$ for all $\tau \in T(A)$.

Then $M(B)$ and $M(B) / B$ have property $P 1$.

Proof. Let $b \in M(B)$ be a full element. Without loss of generality, we may assume that $0 \leq b \leq 1$. Let $\left\{e_{i j}\right\}$ be a system of matrix units for $\mathscr{K}$ and set $E_{n}=\sum_{k=1}^{n} e_{i i}$. Then $E_{n} b E_{n}$ converges to $b$ in the strict topology. Furthermore $b^{1 / 2} E_{n} b^{1 / 2}$ increasingly converges to $b$ in the strict topology.

Since $b$ is full, there are $x_{1}, x_{2}, \ldots, x_{m} \in M(B)$ such that

$$
\sum_{k=1}^{m} x_{i}^{*} b x_{i}=1 \text {. }
$$

Let $\tau \in T(A)$ be a tracial state. We extend $\tau$ to $B_{+}$and then to $M(B)_{+}$in a usual way. Let $T$ be the set of all (densely defined) traces on $M(B)_{+}$whose restrictions to $A$ are tracial states. With the usual weak *-topology, $T$ is a compact convex set.

Because $b^{1 / 2} x_{i}^{*} x_{i} b^{1 / 2} \leq\left\|x_{i}\right\|^{2} b$, one has

$$
\tau\left(x_{i}^{*} b x_{i}\right)=\tau\left(b^{1 / 2} x_{i}^{*} x_{i} b^{1 / 2}\right) \leq\left\|x_{i}\right\|^{2} \tau(b)
$$

for all $\tau \in T(A)$ Therefore

$$
\sum_{i=1}^{m} \tau\left(x_{i}^{*} b x_{i}\right) \leq\left(\sum_{i=1}^{m}\left\|x_{i}\right\|^{2}\right) \tau(b)
$$

for all $\tau \in T(A)$. Since $\tau(1)=\infty$, it follows that $\tau(b)=\infty$. Because $b^{1 / 2} E_{n} b^{1 / 2} \nearrow$ $b$ and because $T$ is compact, by Dini's theorem, we have $\tau\left(b^{1 / 2} E_{n} b^{1 / 2}\right) \rightarrow \infty$ uniformly on $T$. Since $\tau\left(E_{n} b E_{n}\right)=\tau\left(b^{1 / 2} E_{n} b^{1 / 2}\right)$ for all $\tau \in T$, we have $\tau\left(E_{n} b E_{n}\right) \nearrow$ $\infty$ uniformly on $T$. There is $n(1) \geq 1$ such that

$$
\tau\left(E_{n(1)} b E_{n(1)}\right)>1+2 d \quad \text { for all } \tau \in T .
$$

Let $A_{1}$ be the hereditary $C^{*}$-subalgebra of $B$ generated by $E_{n(1)} b E_{n(1)}$. It follows from assumption (2) that there is a projection $p_{1} \in A_{1}$ such that $\tau\left(p_{1}\right)>1+d$ for all $\tau \in T$. Thus there is $v_{1} \in B$ such that $v_{1}^{*} v_{1} \leq p_{1}$ and $v_{1} v_{1}^{*}=E_{1}$. There are nonnegative continuous functions $f, g \in C_{0}((0,2\|b\|]$ such that $g f=f$ and

$$
\left\|f\left(E_{n(1)} b E_{n(1)}\right) p_{1} f\left(E_{n(1)} b E_{n(1)}\right)-p_{1}\right\|<\frac{1}{4} .
$$


It follows by [Effros 1981, A8] that there is a projection

$$
q_{1} \in \overline{f\left(E_{n(1)} b E_{n(1)}\right) B f\left(E_{n(1)} b E_{n(1)}\right)}
$$

such that $q_{1}$ is unitarily equivalent to $p_{1}$. We conclude that $g\left(E_{n(1)} b E_{n(1)}\right) q_{1}=q_{1}$, since $g f=f$. By functional calculus, we see that there is $f_{1} \in A_{1}$ such that

$$
f_{1} E_{n(1)} b E_{n(1)} f_{1}=g .
$$

Thus we obtain $z_{1} \in E_{n(1)} B E_{n(1)}$ such that

$$
z_{1}^{*} b z_{1}=z_{1}^{*} E_{n(1)} b E_{n(1)} z_{1}=E_{1} .
$$

Note that $\tau\left(\left(1-E_{n(1)}\right) b E_{n(1)}\right)=\tau\left(b E_{n(1)}\left(1-E_{n(1)}\right)\right)=0$. It follows that

$$
\tau\left(\left(1-E_{n(1)}\right) b\left(1-E_{n(1)}\right)\right)=\tau\left(\left(1-E_{n(1)}\right) b\right) .
$$

Since $\tau\left(E_{n(1)} b E_{n(1)}\right)<\infty$ for all $\tau \in T$, we conclude that

$$
\tau\left(\left(1-E_{n(1)}\right) b\left(1-E_{n(1)}\right)\right)=\infty \quad \text { for all } \tau \in T .
$$

By the earlier argument we obtain $n(2)>n(1)$ and $z_{2} \in\left(E_{n(2)}-E_{n(1)}\right) B\left(E_{n(2)}-E_{n(1)}\right)$ such that

$$
z_{2}^{*} b z_{2}=z_{2}^{*}\left(E_{n(2)}-E_{n(1)}\right) b\left(E_{n(2)}-E_{n(1)}\right) z_{2}=E_{2}-E_{1} .
$$

Continuing this fashion, we obtain a sequence $\{n(k)\}$ with $n(k+1)>n(k)$ and $z_{k} \in\left(E_{n(k+1)}-E_{n(k)}\right) B\left(E_{n(k+1)}-E_{n(k)}\right)$ such that

$$
z_{k}^{*} b z_{k}^{*}=z_{k}^{*}\left(E_{n(k+1)}-E_{n(k)}\right) b\left(E_{n(k+1)}-E_{n(k)}\right) z_{k}=E_{k+1}-E_{k},
$$

for $k=1,2, \ldots$ Hence $z=\sum_{k=1}^{\infty} z_{k} \in M(B)$, since the sum converges in the strict topology. Furthermore,

$$
z^{*} b z=1 \text {. }
$$

This shows that $M(B)$ has property $\mathrm{P} 1$. By Corollary 3.2, $M(B) / B$ also has property P1.

Corollary 3.6. Let $A$ be a unital $A F$-algebra and take $B=A \otimes \mathcal{K}$. Then $M(B)$ and $M(B) / B$ have property $P 1$.

Proof. Clearly $A$ satisfies (1) in Theorem 3.5 with any $d>0$. To see that it satisfies (2), we let $1 \geq b \geq 0$ be an element in $M_{n}(A)$ such that $\tau(b)>\alpha+d$ for all $\tau \in T$. Set $C=\overline{b M_{n}(A) b}$ and let $\left\{e_{n}\right\}$ be an approximate identity for $C$ consisting of projections. Then $\left\|e_{n} b e_{n}-b\right\| \rightarrow 0$ as $n \rightarrow \infty$. Since $0 \leq b \leq 1$, it follows that $\tau\left(e_{n}\right)>\alpha+d$ for some $n>0$ and all $\tau \in T$.

The proof of the corollary implies the following: 
Corollary 3.7. Let $A$ be a unital separable $C^{*}$-algebra with real rank zero for which $T(A) \neq \varnothing$ and which satisfies (1) in Theorem 3.5. Then $M(B)$ and $M(B) / B$ have property $P 1$, where $B=A \otimes K$.

Corollary 3.8. Let $B=A \otimes \mathscr{K}$, where $A$ is a unital simple $C^{*}$-algebra with real rank zero, stable rank one and weakly unperforated $K_{0}(A)$. Then both $M(B)$ and $M(B) / B$ have property $P 1$.

Corollary 3.9. Let $A=C(X)$, where $X$ is a compact Hausdorff space with finite covering dimension $d$. Then $M(A \otimes \mathscr{K})$ and $M(A \otimes \mathscr{K}) /(A \otimes \mathscr{K})$ have property $P 1$.

Proof. Suppose e, $f \in A \otimes \mathscr{K}$ are projections. We may assume that $e, f \in M_{n}(C(X))$ for some integer $n>0$. Suppose that $\tau(e)>\tau(f)+d+1$ for all $t \in T(A)$. It follows that for each $x \in X$, the rank of $e(x)$ is greater than $d+1+$ the rank of $f(x)$. It follows from [Husemoller 1966, 8.1.2 and 8.1.6] (see [Blackadar 1998, 6.10.3(d)]) that $f \lesssim e$. So Theorem 3.5(1) holds (for $(d+1) / 2$ ).

For (2), let $1 \geq b \geq 0$ be an element in $M_{k}(C(X))$ for which $\tau(b)>\alpha+(d+1)$. Let $f_{n}$ be as in (1-1). For some large $n$, we have $\tau\left(f_{n}(b)\right)>\alpha+(d+1)$ for all $\tau \in T(A)$. Thus, for each $\xi \in X$, the rank of $f_{n}(b)(\xi)$ is at least $\alpha+(d+1)$. By [Blackadar et al. 1991, Lemma C], there is a projection $e \in \overline{b M_{k}(A) b}$ such that the rank of $e(\xi)$ is greater than $\alpha$ for all $\xi \in X$. It follows that $\tau(e)>\alpha$ for all $\tau \in T(A)$.

To discuss property $\mathrm{P} 2$, we begin with an easy observation:

Proposition 3.10. Let $B$ be a unital $C^{*}$-algebra having property $P 2$. For any integer $n>0$, there are $s_{11}, s_{22}, \ldots, s_{n n}$ such that $1_{B} \geq \sum_{i=1}^{n} s_{i i}$ and there exists an isometry $Z \in B$ such that $Z Z^{*}=e_{11}$. Moreover:

(1) If for some $n \geq 2,1_{B}=\sum_{i=1}^{n} s_{i i}$, then there exists a unital embedding from $\mathrm{O}_{n}$ to $B$.

(2) There is a unital embedding from $\mathrm{O}_{\infty}$ to $B$.

(3) There exists a full embedding $j: \mathrm{O}_{2} \rightarrow B$.

Conversely, if there is a unital embedding of $\mathrm{O}_{\infty}$ in $B$, then $B$ has property $P 2$. Furthermore, if $B$ admits a full embedding from $\mathrm{O}_{2}$, then $B$ has property $P 2$.

Proposition 3.11. (1) Let $A$ be a unital $C^{*}$-algebra and $B=A \otimes \mathscr{K}$. Then $M(B)$ and $M(B) / B$ has property $P 2$.

(2) Let $A$ be a nonunital $\sigma$-unital simple $C^{*}$-algebra which has continuous scale. Then $M(A) / A$ has property $P 2$

(3) Let $A$ be a unital purely infinite simple $C^{*}$-algebra and $B=C_{0}(X, A)$, where $X$ is a locally compact Hausdorff space. Then $M(B)$ and $M(B) / B$ have property $P 2$. 
Proof. For (3), we note there is a unital embedding from $O_{\infty}$ to $A$ and the constant maps from $X$ into $A$ are in $C^{b}(X, A)=M(B)$.

Now we turn to property P3. Every unital purely infinite simple $C^{*}$-algebra has property P3, by [Lin 2005b, 2.6]. Therefore, if $B$ is a nonunital but $\sigma$-unital simple $C^{*}$-algebra with continuous scale, then $M(B) / B$ has property $\mathrm{P} 3$.

Proposition 3.12. Let $B$ be a unital $C^{*}$-algebra having property $P 1$. Suppose that $0 \leq a, b \leq 1$, where $a b=a$ and $a$ is full. Then there exists $x \in B$ with $\|x\| \leq 1$ and

$$
x^{*} b x=1 .
$$

Note that the proposition includes the case that $a$ is a full projection.

Proof. There is $z \in B$ such that $z^{*} a z=1$. Then $a^{1 / 2} z z^{*} a^{1 / 2}=p$ must be a projection. Moreover, $p \in \operatorname{Her}(a)$. Therefore $p b=p$. Put $v=a^{1 / 2} z$. Then $v^{*} v=1$ and $v v^{*}=p$. In particular, $\|v\|=1$. Now

$$
1 \geq\|b\| v^{*} v \geq v^{*} b v \geq v^{*} p v=1 .
$$

We conclude that $v^{*} b v=1$.

Proposition 3.13. Let $A$ be a unital $C^{*}$-algebra and set $B=A \otimes \mathcal{K}$. Then $M(B) / B$ has property $P 3$.

Proof. Let $\pi: M(B) \rightarrow M(B) / B$ be the quotient map and $D$ a separable $C^{*}$ algebra. Let $\left\{e_{i, j}\right\}$ be a system of matrix units for $\mathscr{K}$. Set $E_{n}=\sum_{i=1}^{n} e_{i, i}$. By [Pedersen 1979, 3.12.14] and the proof of [Lin 2001, 5.5.3], there is a sequence $\left\{e_{n}\right\} \subset \operatorname{Conv}\left\{E_{k}: k=1,2, \ldots\right\}$ such that

$$
e_{n+1} e_{n}=e_{n} \quad \text { and } \quad\left\|e_{n} a-a e_{n}\right\| \rightarrow 0 \quad \text { as } n \rightarrow \infty
$$

for all $a \in D$.

Suppose that $e_{n}=\sum_{i=1}^{k(n)} \alpha_{i} E_{i}$, where the $\alpha_{i}$ are nonnegative scalars such that $\sum_{i=1}^{k(n)} \alpha_{i}=1$. There exist $0 \leq \beta_{j} \leq 1$ such that $e_{n}=\sum_{i=1}^{k(n)} \beta_{j} e_{j j}$. Since, for each $i$,

$$
\left\|e_{m} e_{i i}-e_{i i}\right\| \rightarrow 0 \quad \text { as } m \rightarrow \infty,
$$

there is $N(n)>0$ such that, for each $m>N(n), e_{m}=\sum_{i=1}^{k(m)} \beta_{i}^{(m)} e_{i i}$ with $\beta_{k(n)+1}>$ $\frac{1}{2}$. It follows that

$$
\begin{aligned}
\left(e_{m}-e_{n}\right) e_{k(n)+1, k(n)+1} & =\left(\sum_{k(n)+1}^{k(m)} \beta_{i}^{(m)} e_{i i}+\sum_{i=1}^{k(n)}\left(\beta_{i}^{(m)}-\beta_{i}^{(n)}\right) e_{i i}\right) e_{k(n)+1, k(n)+1} \\
& =\left(\sum_{k(n)+1}^{k(m)} \beta_{i}^{(m)} e_{i i}\right) e_{k(n)+1, k(n)+1}=\beta_{i}^{(m)} e_{k(n)+1, k(n)+1}
\end{aligned}
$$


By passing to a subsequence if necessary we may as well assume that

$$
\left(e_{n+1}-e_{n}\right) e_{k(n)+1, k(n)+1}=\lambda_{n} e_{k(n)+1, k(n)+1}
$$

for some $\lambda_{n}>\frac{1}{2}$. Now let $F \subset \mathbb{N}$ be an infinite subset. Then

$$
b_{F}=\sum_{n \in F}\left(e_{n+1}-e_{n}\right) \geq \frac{1}{2} \sum_{n \in F} e_{k(n)+1, k(n)+1} .
$$

Thus $b_{F}$ is a full positive element in $M(B)$. Suppose that $\left\{F_{n}\right\}$ is a sequence of infinite subsets of $\mathbb{N}$. By Proposition 3.12, the image $\pi\left(\left\{\sum_{j \in F_{n}} e_{k(j)+1, k(j)+1}\right\}\right)$ is full in $l^{\infty}(M(B) / B)$. So $\left\{\pi\left(b_{F_{n}}\right)\right\}$ is full in $l^{\infty}(M(B) / B)$.

By (3-1), $\pi\left(b_{F}\right)$ commutes with $\pi(d)$ for each $d \in D$. Also by (3-1)

$$
\left(e_{n+1}-e_{n}\right)\left(e_{m+1}-e_{m}\right)=0 \text { if }|n-m| \geq 2 .
$$

It follows that $b_{F} b_{F^{\prime}}=0$ if $|n-m| \geq 2$ for any $n \in F$ and any $m \in F^{\prime}$. Note that one may write $b_{F}=\sum_{n \in S(F)} \lambda_{n} e_{n, n}$, where each $0<\lambda_{n} \leq 1$ is a positive number and $S(F)$ is an infinite subset of $\mathbb{N}$.

It is easy to find a family of (disjoint) infinite subsets $\left\{F_{i, j}: i, j=1,2, \ldots\right\}$ of $\mathbb{N}$ such that $|n-m| \geq 2$ for any $n \in S_{i, j}$ and any $m \in S_{i^{\prime}, j^{\prime}}$, if $i \neq i^{\prime}$ or $j \neq j^{\prime}$. Define $S_{i, j}=S\left(F_{i, j}\right)$ as above. We note that $S_{i, j} \cap S_{i^{\prime}, j^{\prime}}=\varnothing$ if $i \neq i^{\prime}$ or $j \neq j^{\prime}$. Write $b_{i, j}$ for $b_{F_{i, j}}$. It follows that $M(B) / B$ has property P3.

\section{Nonstable cases}

In [Kirchberg and Rørdam 2000] the notion of purely infinite $C^{*}$-algebras was extended to nonsimple $C^{*}$-algebras. Let $C_{1}$ be a unital $C^{*}$-algebra and $C_{2}$ be a unital separable purely infinite simple $C^{*}$-algebra. Then $C_{1} \otimes C_{2}$ is purely infinite [Kirchberg and Rørdam 2000, 4.5]. Therefore, for any unital $C^{*}$-algebra $C$ the $C^{*}$-algebra $B=C \otimes \mathrm{O}_{\infty}$ has properties $\mathrm{P} 1$ and $\mathrm{P} 2$ as well as $\mathrm{P} 3$.

Proof of Proposition 2.2. From the preceding paragraph, we know that $B$ is purely infinite, and so has properties $\mathrm{P} 1$ and $\mathrm{P} 2$. Let $A$ be a separable $C^{*}$-subalgebra of $B$. There is a separable $C^{*}$-subalgebra $C_{0} \subset C$ such that $A \subset C_{0} \otimes C_{1}$. It follows from [Kirchberg and Phillips 2000] that $C_{1} \otimes \mathcal{O}_{\infty} \cong C_{1}$. By [Rørdam 2002, 7.2.6] and [Kirchberg and Phillips 2000, 3.12], there is a sequence of unital monomorphisms $\phi_{n}: \mathbb{O}_{\infty} \rightarrow C_{0} \otimes C_{1}$ such that

$$
\lim _{n \rightarrow \infty}\left\|\phi_{n}(x) a-a \phi_{n}(x)\right\|=0 \quad \text { for all } a \in C_{0} \otimes \mathscr{C}_{1} .
$$

Let $\left\{e_{k}\right\}$ be a sequence of nonzero mutually orthogonal projections in $\mathrm{O}_{\infty}$. Define $a_{n}^{(i)}=\phi_{n}\left(e_{i}\right), n, i=1,2, \ldots$ One checks that $a_{n}^{(i)}$ satisfies the requirements for property $\mathrm{P} 3$. 
There are $\sigma$-unital but nonstable separable $C^{*}$-algebras $B$ for which the corona $C^{*}$-algebra $M(B) / B$ has properties $\mathrm{P} 1$ and $\mathrm{P} 2$ as well as $\mathrm{P} 3$. For example, when $B$ has continuous scale, $M(B) / B$ is a purely infinite simple $C^{*}$-algebra [Lin 2004b]. So in those cases $M(B) / B$ has all three properties. There are other nonstable separable $C^{*}$-algebras $B$ for which $B$ has properties P1, P2 and P3.

To make a point, we will present a very simple example of a nonstable $\sigma$-unital $C^{*}$-algebra $B$ for which $M(B) / B$ is not simple but both $M(B)$ and $M(B) / B$ have properties $\mathrm{P} 1, \mathrm{P} 2$ and $M(B) / B$ has $\mathrm{P} 3$. Many such examples can be constructed.

Proposition 4.3 is not needed in Example 4.4 but will be used again later.

Lemma 4.1. Let $A$ be a unital $C^{*}$-algebra and let $0 \leq a \leq 1$ be an element in $A$. Suppose that there is $x \in A$ such that $x^{*} a x=1$. Then there is $N>0$, depending on $\|x\|$ but not on $A$ or a, for which there is $y \in A$ with $\|y\| \leq 1$ such that

$$
y^{*} f_{N}(a) y=1 .
$$

In particular, $f_{N}(a)$ is full, where $f_{N}$ is as defined in (1-1).

Proof. Let $q=a^{1 / 2} x x^{*} a^{1 / 2}$. Then $q$ is a projection. There exists $k>0$ depending on $\|x\|$ such that

$$
\left\|f_{k}(t) t^{1 / 2}-t^{1 / 2}\right\|<\frac{1}{16\|x\|^{2}} \quad \text { for all } t \in[0,1],
$$

where $f_{k}$ is as in (1-1). Then

$$
\left\|f_{k}(a) q-q\right\|=\left\|\left(f_{k}(a) a^{1 / 2}-a^{1 / 2}\right) x^{*} x a^{1 / 2}\right\|<\frac{1}{16} .
$$

It follows from [Effros 1981, A8] that there is a projection $p \in \overline{f_{k}(a) A f_{k}(a)}$ such that

$$
\|q-p\|<\frac{1}{2} .
$$

Thus there exists $w \in A$ such that $w^{*} w=1$ and $w w^{*}=p$. Choose $N=k+1$. Then $f_{N}(a) q=q$, showing that

$$
w^{*} f_{N}(a) w=1 .
$$

Lemma 4.2. Let $A$ be a unital $C^{*}$-algebra and let $a \in A$ with $0 \leq a \leq 1$ be a full element. Suppose that there are $x_{1}, x_{2}, \ldots, x_{m} \in A$ such that

$$
\sum_{i=1}^{m} x_{i}^{*} a x_{i}=1 \text {. }
$$

Set $r=\sum_{i=1}^{m}\left\|x_{i}\right\|^{2}$. Suppose also that $1_{M_{m}(A)} \lesssim 1$. Then there exists an integer $N>0$, depending on $r$ but not on A on a, such that $f_{N}(a)$ is full. Moreover, there 
are $y_{1}, y_{2}, \ldots, y_{m} \in A$ such that $\sum_{i=1}^{m}\left\|y_{i}\right\|^{2} \leq 1$ and

Proof. Let

$$
\sum_{i=1}^{m} y_{i}^{*} f_{N}(a) y_{i}=1 \text {. }
$$

$$
X=\left(\begin{array}{cccc}
x_{1} & x_{2} & \cdots & x_{m} \\
0 & 0 & \cdots & 0 \\
\vdots & \vdots & \ddots & \vdots \\
0 & 0 & \cdots & 0
\end{array}\right) \quad \text { and } \quad b=\left(\begin{array}{cccc}
a & 0 & \cdots & 0 \\
0 & a & \cdots & 0 \\
\vdots & \vdots & \ddots & \vdots \\
0 & 0 & \cdots & a
\end{array}\right)
$$

Since $1_{M_{m}(A)} \lesssim 1$, one obtain $Y \in M_{m}(A)$ with $\|Y\|=1$ and $Y^{*} \operatorname{diag}(1,0, \ldots, 0) Y=$ $1_{M_{m}(A)}$. Note that $0 \leq b \leq 1$ and $X b X^{*}=\operatorname{diag}(1,0, \ldots, 0)$. Thus

$$
Y^{*} X b X^{*} Y=1_{M_{m}(A)} .
$$

We compute that $\left\|X^{*} Y\right\| \leq r^{1 / 2}$. It follows from Lemma 4.1 that there exist $N>0$ and $z \in M_{m}(A)$ with $\|z\| \leq 1$ such that

$$
z^{*} f_{N}(b) z=1_{M_{m}(A)} .
$$

So $Y z^{*} f_{N}(b) z Y^{*}=1$. An easy computation shows that there are $y_{1}, y_{2}, \ldots, y_{n} \in A$ such that $\sum_{i=1}^{m}\left\|y_{i}\right\|^{2} \leq 1$ and

$$
\sum_{i=1}^{m} y_{i}^{*} f_{N}(a) y_{i}=1 .
$$

Proposition 4.3. Let $\left\{A_{n}\right\}$ be a sequence of unital $C^{*}$-algebras having property $P 1$. Then $l^{\infty}\left(\left\{A_{n}\right\}\right)$ also has property $P 1$.

Proof. Let $a=\left\{a_{n}\right\}$ be a full element in $l^{\infty}\left(\left\{A_{n}\right\}\right)$ such that $0 \leq a \leq 1$. (Note that full elements of $l^{\infty}\left(\left\{A_{n}\right\}\right)$ cannot be in $c_{0}\left(\left\{A_{n}\right\}\right)$.) By Lemma 4.2, there exists $N>0$ for which $f_{N}(a)$ is full. For each $n$, there exists $x_{n} \in A_{n}$ such that $x_{n}^{*} f_{N}\left(a_{n}\right) x_{n}=1$. Note that $f_{N+1}\left(a_{n}\right) f_{N}(a)=f_{N}(a)$. It follows from Proposition 3.12 that, for each $n$, there is $y_{n} \in A$ with $\left\|y_{n}\right\| \leq 1$ such that

$$
y_{n}^{*} f_{N+1}(a) y_{n}=1 .
$$

Put $y=\left\{y_{n}\right\}$. Then $y \in l^{\infty}\left(\left\{A_{n}\right\}\right)$. It is clear that there is $g \in C_{0}((0,1])_{+}$such that

$$
\left\|g(a) \operatorname{ag}(a)-f_{N+1}(a)\right\|<\frac{1}{4} .
$$

Then

$$
\left\|y^{*} g(a) \operatorname{ag}(a) y-1\right\|=\left\|y^{*}\left(g(a) \operatorname{ag}(a)-f_{N}(a)\right) y\right\| \leq \frac{1}{4} .
$$

Hence there is $z \in l^{\infty}\left(\left\{A_{n}\right\}\right)$ with $\|z\|<\frac{4}{3}$ such that

$$
z^{*} y^{*} g(a) \operatorname{ag}(a) y z=1 .
$$


This proposition is not required in the following example. However it will be used in Lemma 6.5.

Example 4.4. Let $A$ be a unital separable amenable purely infinite simple $C^{*}$ algebras. Denote by $B=c_{0}(A)$. Then $M(B)=l^{\infty}(A)$. Put $q_{\infty}(A)=l^{\infty}(A) / c_{0}(A)$. So $M(B) / B=q_{\infty}(A)$.

(1) $M(B)$ and $M(B) / B$ has properties $\mathrm{P} 1$ and $\mathrm{P} 2$.

(2) $M(B) / B$ has property $\mathrm{P}$.

Claim (1) is obvious (it also follows from Proposition 4.3). In fact, if $C=$ $C_{0}((0,1), A)$, then $M(C)$ and $M(C) / C$ also have properties $\mathrm{P} 1$ and $\mathrm{P} 2$. This can be proved rather easily.

To see (2), let $D$ be a separable $C^{*}$-subalgebra of $M(B)$. Suppose that $x^{(1)}=$ $\left\{x_{n}^{(1)}\right\}, x^{(2)}=\left\{x_{n}^{(2)}\right\}, \ldots, x^{(k)}=\left\{x_{n}^{(k)}\right\}, \ldots$ is a dense sequence in the unit ball of $D$. Using the fact that $A \otimes 0_{\infty} \cong A$ [Kirchberg and Phillips 2000, Theorem 3.15], we obtain a sequence of homomorphisms $\phi_{n}: \mathrm{O}_{\infty} \rightarrow A$ such that

$$
\lim _{n \rightarrow \infty}\left\|\phi_{n}(b) a-a \phi_{n}(b)\right\|=0
$$

for all $a \in A$ and $b \in \mathcal{O}_{\infty}$. Let $e_{1} \in \mathcal{O}_{\infty}$ be a proper projection. There is an integer $n(1)>0$ such that

$$
\left\|\phi_{n(1)}\left(e_{1}\right) x_{1}^{(1)}-x_{1}^{(1)} \phi_{n(1)}\left(e_{1}\right)\right\|<\frac{1}{2} .
$$

There is a projection $e_{2} \in \mathbb{O}_{\infty}$ such that $e_{1} e_{2}=e_{2} e_{1}=0$ and $1>e_{1}+e_{2}$. There is $n(2)>0$ such that

$$
\left\|\phi_{n(2)}\left(e_{j}\right) x_{l}^{(i)}-x_{l}^{(i)} \phi_{n(2)}\left(e_{j}\right)\right\|<\frac{1}{4} \quad \text { for } i, j, l=1,2 .
$$

Continuing in this fashion, we obtain a sequence of mutually orthogonal nonzero projections $\left\{e_{m}\right\} \subset O_{\infty}$ and a subsequence $\{n(m)\}$ such that

$$
\left\|\phi_{n(m)}\left(e_{j}\right) x_{l}^{(i)}-x_{l}^{(i)} \phi_{n(m)}\left(e_{j}\right)\right\|<2^{-m} \text { for } i, j, l=1,2, \ldots, m .
$$

Put $p^{(j)}=\left\{\phi_{n(m)}\left(e_{j}\right)\right\} \in l^{\infty}(A)$, for $j=1,2, \ldots$. Then $p_{m}^{(i)} p_{m}^{(j)}=0$ if $i \neq j$. Moreover,

$$
\left\|\pi\left(p^{(j)}\right) \pi\left(\left\{x^{(i)}\right\}\right)-\pi\left(\left\{x^{(i)}\right\}\right) \pi\left(p^{(j)}\right)\right\|=0 .
$$

This implies that

$$
\pi\left(p^{(j)}\right) \pi(d)=\pi(d) \pi\left(p^{(j)}\right) .
$$

Put $a_{n}^{(j)}=p^{(j)}, j=1,2, \ldots$ This shows that $M(B) / B$ has property P3.

It is clear, in fact, that $l^{\infty}\left(\left\{A_{n}\right\}\right) / c_{0}\left(\left\{A_{n}\right\}\right)$ has property P3 if each $A_{n}$ is a unital purely infinite simple $C^{*}$-algebra. 


\section{Amenable contractive completely positive linear maps}

Lemma 5.1 ([Akemann et al. 1986, 2.3]; see also [Lin 2001, 5.3.2]). Let $A$ be a separable $C^{*}$-algebra and $\psi: A \rightarrow \mathbb{C}$ a pure state. Denote by $\psi$ also the extension of $\psi$ to $\tilde{A}$ and put $L=\left\{a \in \tilde{A}: \psi\left(a^{*} a\right)=0\right\}$. For any $\varepsilon>0$ and any finite subset $\mathscr{F} \subset A$, there exist $z_{1}, z_{2}, z_{3} \in \tilde{A}_{+}$such that $\left\|z_{i}\right\|=1$ and $z_{i} \notin L$ for $i=1,2,3$, $z_{i+1} z_{i}=z_{i}$ for $i=1,2$, and

$$
\left\|z_{i}(\phi(a)-a) z_{i}\right\|<\frac{1}{2} \varepsilon \quad \text { for } i=1,2,3 \text { and all } a \in \mathscr{F} .
$$

Moreover, if $\left\{e_{n}\right\}$ is an approximate identity for $A$, then, for some large $N$,

$$
\left\|e_{n} z_{i} e_{n}(\phi(a)-a) e_{n} z_{i} e_{n}\right\|<\varepsilon \quad \text { and } e_{n} z_{i} e_{n} \notin L
$$

for all $a \in \mathscr{F}$ and all $n \geq N$.

Proof. To simplify notation, we assume that $\mathscr{F}$ is a subset of the unit ball of $A$. Let

$$
N=\{a \in \tilde{A}: \phi(a)=0\} .
$$

Note that $L$ is a closed left ideal. Let $C$ be the hereditary $C^{*}$-subalgebra given by $L \cap L^{*}$. As in the proof of [Lin 2001, 5.3.2], we have $z_{1}, z_{2}, z_{3}, \in \tilde{A}$ with $\left\|z_{i}\right\|=1$ $(i=1,2,3)$ such that $z_{i} \notin L, z_{i+1} y_{i}=z_{i}, i=1,2,3$, and

$$
\left\|z_{i}(\psi(a)-a) z_{i}\right\|<\frac{1}{2} \varepsilon, \quad i=1,2,3 .
$$

Let $\left\{e_{n}\right\}$ be an approximate identity for $A$ such that $e_{n} e_{n+1}=e_{n}$ for all $n$. Note that $z_{i}$ has the form $\lambda_{i} 1_{B}+y_{i}^{\prime}$, where $y_{i}^{\prime} \in A$ and $\lambda_{i} \in \mathbb{C}, i=1$, 2. Choose a large $n$ such that

$$
\left\|e_{k} a-a e_{k}\right\|<\frac{1}{4} \varepsilon, \quad\left\|e_{k} a-a\right\|<\frac{1}{4} \varepsilon \quad \text { and } \quad\left\|e_{k} z_{i}-z_{i} e_{k}\right\|<\frac{1}{4} \varepsilon
$$

for all $a \in \mathscr{F} \cup\left\{z_{1} a z_{1}, z_{2} a z_{2}, z_{3} a z_{3}: a \in \mathscr{F}\right\}$ and for all $k \geq n$. Let $y_{i}=e_{n} z_{i} e_{n}$. Then, for $n \geq N$,

$$
\left\|y_{i}(\psi(a)-a) y_{i}\right\|<\frac{1}{4} \varepsilon+\left\|e_{n}^{2} z_{i}(\psi(a)-a) z_{i} e_{n}^{2}\right\|<\varepsilon \quad \text { for all } a \in \mathscr{F} .
$$

The following is folklore.

Lemma 5.2. Let $A$ be a $C^{*}$-subalgebra of $B$ and take $a \in A_{+}$. Denote by $C$ the hereditary $C^{*}$-subalgebra of $B$ generated by $a$. Then, for any approximate identity $\left\{e_{n}\right\}$ of $A$, the sequences $\left\|e_{n} b-b\right\|$ and $\left\|b e_{n}-b\right\|$ converge to 0 as $n \rightarrow \infty$, for all $b \in C$.

Proof. There exists a sequence of positive functions $f_{n} \in C_{0}(\operatorname{sp}(a))$ with $0 \leq f_{n} \leq 1$ such that $\left\{f_{n}(a)\right\}$ forms an approximate identity for $C$. Fix an element $b \in C$. For any $\varepsilon>0$, there is $f_{k}$ such that

$$
\left\|f_{k}(a) b-b\right\|<\frac{1}{4} \varepsilon \quad \text { and } \quad\left\|b f_{k}(a)-b\right\|<\frac{1}{4} \varepsilon .
$$


Choose an integer $N>0$ such that

$$
\left\|e_{n} f_{k}(a)-f_{k}(a)\right\|<\frac{\varepsilon}{4(\|b\|+1)} \quad \text { for all } n \geq N .
$$

Then

$$
\begin{aligned}
\left\|e_{n} b-b\right\| & \leq\left\|e_{n} b-e_{n} f_{k}(a) b\right\|+\left\|e_{n} f_{k}(a) b-f_{k}(a) b\right\|+\left\|f_{k}(a) b-b\right\| \\
& <\frac{1}{4} \varepsilon+\|b\|\left(\frac{\varepsilon}{4(\|b\|+1)}\right)+\frac{1}{4} \varepsilon=\frac{3}{4} \varepsilon<\varepsilon .
\end{aligned}
$$

Lemma 5.3. Let $B$ be a unital $C^{*}$-algebra that has the property $P 1$. Let $A$ be a separable $C^{*}$-algebra and let $I$ be an ideal of $A$. Suppose that $j: A \rightarrow B$ is an embedding such that $j(a)$ is a full element of $B$ for all $a \notin I$. Then, for any pure state $\phi: A \rightarrow \mathbb{C} 1_{B} \subset B$ which vanishes on $I$, any finite subset $\mathscr{F} \subset A$, and any $\varepsilon>0$, there is a partial isometry $V \in B$ such that

$$
\left\|\phi(a)-V^{*} j(a) V\right\|<\varepsilon \quad \text { for } a \in \mathscr{F}, \quad V^{*} V=1_{B} \quad \text { and } \quad V V^{*} \in \operatorname{Her}(j(A)) .
$$

Proof. To simplify notation, we identify $A$ with $j(A)$. Fix $0<\varepsilon<\frac{1}{2}$. By Lemma 5.1, there are $z_{1}, z_{2}, z_{3} \in \tilde{A}_{+}$such that $\left\|z_{i}\right\|=1$ and $z_{i} \notin L$ for $i=1,2,3, z_{i+1} z_{i}=z_{i}$ for $i=1,2$, and

$$
\left\|\phi(a) z_{i}^{2}-z_{i} j(a) z_{i}\right\|<\frac{1}{4} \varepsilon \quad \text { for } i=1,2 \text { and all } a \in \mathscr{F} .
$$

Note that $L=\left\{a \in \tilde{A}: \psi\left(a^{*} a\right)=0\right\}$. Therefore $I \subset L \cap L^{*} \subset L$. Let $\left\{e_{n}\right\}$ be an approximate identity for $A$ such that $e_{n} e_{n+1}=e_{n}, n=1,2, \ldots$ Let $N$ be an integer as in Lemma 5.1, such that

$$
\left\|\phi(a)\left(e_{n} z_{i} e_{n}\right)^{2}-e_{n} z_{i} e_{n} j(a) e_{n} z_{i} e_{n}\right\|<\frac{1}{2} \varepsilon, \quad \text { for } i=1,2,3 .
$$

Put $y_{1}=e_{N} z_{1} e_{N}$. We may assume that $y_{1} \notin L$. By the assumption, $y_{1}$ is full. Because $B$ has property P1, there exists $x \in B$ such that $x^{*} y_{1}^{2} x=1_{B}$. Put $v_{1}=y_{1} x$. Then $v_{1}^{*} v_{1}=1_{B}$ and $v_{1} v_{1}^{*}=p_{1}$ is a projection. Note that $p_{1} \in \operatorname{Her}\left(y_{1}\right)$. There is a projection in $q_{1} \in \operatorname{Her}\left(z_{1}^{1 / 2} e_{N} z_{1}^{1 / 2}\right)$ such that $q_{1}$ is equivalent to $p_{1}$. Therefore there is a partial isometry $w_{1} \in B$ such that $w_{1}^{*} q_{1} w_{1}=1_{B}$ and $w_{1} w_{1}^{*}=q_{1}$. Since $z_{2}^{2} z_{1}=z_{1}, z_{2}^{2} q_{1}=q_{1}$. By applying Lemma 5.2, one can choose a large integer $k>N$ such that, for all $n \geq k$,

$$
\left\|e_{n} q_{1}-q_{1}\right\|<\frac{1}{32} \varepsilon \quad \text { and } \quad\left\|e_{n} z_{i}-z_{i} e_{n}\right\|<\frac{1}{32} \varepsilon \quad \text { for } i=1,2,3 .
$$

Thus

$$
\left\|\left(e_{k} z_{2} e_{k}\right)^{2} q_{1}-q_{1}\right\|=\left\|e_{k} z_{2} e_{k}^{2} z_{2} e_{k} q_{1}-q_{1}\right\|<\frac{8}{32} \varepsilon=\frac{1}{4} \varepsilon .
$$

Put $y_{2}=e_{k} z_{2} e_{k}$. Then one estimates

$$
\left\|w_{1}^{*} y_{2}^{2} w_{1}-1\right\|=\left\|w_{1}^{*} q_{1} y_{2}^{2} q_{1} w_{1}-w_{1}^{*} q_{1} w_{1}\right\|<\frac{1}{2} \varepsilon .
$$


Thus there is $s \in \operatorname{Her}\left(z_{1}^{1 / 2} e_{N} z_{1}^{1 / 2}\right)_{+} \subset B_{+}$such that $\|s\| \leq \frac{1}{1-\frac{1}{2} \varepsilon}$ and

$$
s^{1 / 2} w_{1}^{*} y_{2}^{2} w_{1} s^{1 / 2}=1 .
$$

Note that

$$
\left\|w_{1} s^{1 / 2}\right\| \leq \sqrt{\frac{1}{1-\frac{1}{2} \varepsilon}}<\sqrt{\frac{2}{2-\frac{1}{2}}}=\sqrt{\frac{4}{3}}=\frac{2 \sqrt{3}}{3} .
$$

Define $V=y_{2} w_{1} s^{1 / 2}$. Note that

$$
V^{*} V=1_{B} \quad \text { and } \quad V V^{*} \in \operatorname{Her}(j(A)) .
$$

Put $y_{3}=e_{k+1} z_{3} e_{k+1}$. Then, by (5-2),

$$
\left\|y_{3} y_{2}-y_{2}\right\|=\left\|e_{k+1}\left(z_{3} e_{k} z_{2}-z_{2}\right) e_{k}\right\|<\frac{1}{32} \varepsilon .
$$

Furthermore, by (5-3) and (5-5),

$$
\left\|y_{3} V-V\right\|=\left\|y_{3} y_{2} w_{1} s^{1 / 2}-y_{2} w_{1} s^{1 / 2}\right\| \leq\left(\frac{1}{32} \varepsilon\right) \frac{2 \sqrt{3}}{3}=\frac{\sqrt{3}}{48} \varepsilon .
$$

We estimate, by applying (5-6), (5-4) and (5-1), that

$$
\begin{aligned}
\left\|\phi(a)-V^{*} a V\right\| & =\left\|\phi(a) V^{*} V-V^{*} a V\right\| \leq \frac{\sqrt{3}}{48} \varepsilon+\left\|\phi(a) V^{*} y_{2}^{2} V-V^{*} y_{2} a y_{2} V\right\| \\
& \leq \frac{\sqrt{3}}{48} \varepsilon+\left\|\phi(a) y_{2}^{2}-y_{2} a y_{2}\right\|<\frac{\sqrt{3}}{48} \varepsilon+\frac{1}{2} \varepsilon<\varepsilon \quad \text { for all } a \in \mathscr{F}_{.}
\end{aligned}
$$

Remark 5.4. If $A$ has a unit, the proof of Lemma 5.3 is almost identical to that of [Lin 2001, 5.3.2], which has its origin in [Akemann et al. 1986]. When $A$ has no unit, the elements $z_{1}, z_{2}, z_{3}$ are not in $A_{+}$but in $\tilde{A}_{+}$. By using an approximate identity $\left\{e_{n}\right\}$, one does have $\left\|y_{3} y_{2}-y_{2}\right\|$ small. However the norm of $x$ could be large and it depends on the choice of $z_{i}$ as well as $N$ as in the proof above. By introducing $q_{1}$, we are able to control the norm of $w_{1} s^{1 / 2}$.

Lemma 5.5. Let $B$ be a unital $C^{*}$-algebra having property $P 1$ and let $A$ be a separable $C^{*}$-algebra. Suppose there exists a sequence of homomorphism $\phi_{n}$ : $A \rightarrow B$ such that $\left\{\phi_{n}(a): n=1,2, \ldots\right\}$ is an orthogonal set in $B$ for all $a \in A$. Let $I$ be an ideal of $A$ such that $\operatorname{ker} \phi_{n} \subset I$ and $\phi_{n}(a)$ is a full element in $B$ for all $a \notin I$ and for all $n$. Then, for any state $\psi: A / I \rightarrow \mathbb{C} 1_{B} \subset B$, any finite subset $\mathscr{F} \subset A$, and any $\varepsilon>0$, there is a partial isometry $V \in B$ and an integer $n$ such that $V^{*} V=1 B$

$$
\left\|\psi \circ \pi(a)-V^{*}\left(\sum_{k=1}^{n} \phi_{k}(a)\right) V\right\|<\varepsilon \quad \text { for } a \in \mathscr{F}_{F} \quad \text { and } \quad V V^{*} \in \operatorname{Her} \sum_{i=1}^{n} \phi_{i}(A),
$$


where $\pi: A \rightarrow A / I$ is the quotient map. If $\psi$ is only assumed to be a nonzero positive linear functional with $\|\psi\| \leq 1$, the conclusion still holds with the difference that $V$ is merely a contraction.

Proof. By the Krein-Milman theorem, we have positive numbers $\alpha_{1}, \alpha_{2}, \ldots, \alpha_{m}$ with $\sum_{i=1}^{m} \alpha_{i}=1$ and pure states $\psi_{1}, \psi_{2}, \ldots, \psi_{m}$ of $A / I$ such that

$$
\left\|\psi \circ \pi(a)-\sum_{i=1}^{m} \alpha_{i} \psi_{i}(a)\right\|<\frac{1}{2} \varepsilon \quad \text { for } a \in \mathscr{F} .
$$

Let $\pi_{n}: A \rightarrow A / \operatorname{ker} \phi_{n}$ and $\gamma_{n}: A / \operatorname{ker} \phi_{n} \rightarrow A / I$ be the quotient maps, for $n=1,2, \ldots$. Note that $\psi_{i} \circ \gamma_{n}$ is a pure state of $A / \operatorname{ker} \phi_{n}$.

By Lemma 5.5, there are $V_{i} \in B$ such that $V_{i}^{*} V_{i}=1_{B}, V_{i} V_{i}^{*} \in \operatorname{Her} \phi_{i}(A)$ and

$$
\left\|\psi_{i} \circ \gamma_{i}\left(\phi_{i}(a)\right)-V_{i}^{*} \phi_{i}(a) V_{i}\right\|<\varepsilon \quad \text { for all } a \in \mathscr{F} \text {. }
$$

(Note that $\psi_{i} \circ \gamma_{i} \circ \phi_{i}=\psi_{i} \circ \pi$.)

Set $V=\sum_{i=1}^{m} \sqrt{\alpha_{i}} V_{i} \in B$. Since $\left\{\phi_{n}(a): n=1,2, \ldots\right\}$ is an orthogonal set for each $a \in A$, we compute that

$$
V^{*} V=\sum_{i=1}^{m} 1_{B}=1_{B} \quad \text { and } \quad V V^{*}=\sum_{i, j} \sqrt{\alpha_{i} \alpha_{j}} V_{i} V_{j}^{*} \in \operatorname{Her} \sum_{i=1}^{m} \phi_{i}(A) .
$$

Moreover

$$
\begin{aligned}
\left\|\psi \circ \pi(a)-V^{*}\left(\sum_{i=1}^{m} \phi_{i}(a)\right) V\right\| \\
\quad=\left\|\psi \circ \pi(a)-\sum_{i=1}^{m} \alpha_{i} V_{i}^{*} \phi_{i}(a) V_{i}\right\| \\
\leq\left\|\psi \circ \pi(a)-\sum_{i=1}^{m} \alpha_{i} \psi_{i}(a)\right\|+\sum_{i=1}^{m} \alpha_{i}\left\|\psi_{i} \circ \pi(a)-V_{i}^{*} \phi_{i}(a) V_{i}\right\| \\
\quad<\frac{1}{2} \varepsilon+\frac{1}{2} \varepsilon=\varepsilon \quad \text { for } a \in \mathscr{F} .
\end{aligned}
$$

To check the last statement of the lemma, note that there is $0<\lambda \leq 1$ such that $\psi(a)=\lambda \cdot g(a)$ for some state $g$ and for all $a \in A$.

Lemma 5.6. Let $A$ be a separable $C^{*}$-algebra and let $B$ be a unital $C^{*}$-algebra having properties $P 1$ and $P 2$. Let $C$ be as described in the definition of Property $P 2$, with $n=k$ (see Proposition 3.10). Suppose that $\phi_{n}: A \rightarrow B$ is a sequence of homomorphisms such that $\left\{\phi_{n}(a): n=1,2, \ldots\right\}$ is an orthogonal set in $B$. Suppose that $I$ is an ideal of $A$ such that $I \supset \operatorname{ker} \phi_{n}$ and $\phi_{n}(a)$ is a full element for all $a \notin I$. Let $\psi: A / I \rightarrow M_{k}(\mathbb{C}) \subset M_{k}(C) \subset B$ be a contractive completely positive linear map. Then, for any finite subset $\mathscr{F} \subset A$ and $\varepsilon>0$, there exists a contraction $V \in B$ 
and an integer $m>0$ such that

$$
\left\|\psi(a)-V^{*}\left(\sum_{i=1}^{m} \phi_{i}(a)\right) V\right\|<\varepsilon \quad \text { for } a \in \mathscr{F} \quad \text { and } \quad V V^{*} \in \operatorname{Her} \sum_{i=1}^{K} \phi_{i}(A),
$$

where $\pi: A \rightarrow A / I$ is the quotient map.

Proof. Write $\psi(a)=\sum_{i=1}^{k} \psi_{i j}(a) \otimes s_{i j}$ for $a \in A$, where $\left\{s_{i j}\right\}$ is a system of matrix units for $M_{n}$ and $\psi_{i j}: A \rightarrow \mathbb{C}$ is linear. Also assume that $s_{i i}$ are as in Property P2 and Proposition 3.10, for $i=1,2, \ldots, k$. Define $\Phi: M_{k}(A) \rightarrow \mathbb{C} \subset C$ by $\Phi\left(\left(a_{i j}\right)_{k \times k}\right)=\sum_{i, j=1} \psi_{i j}\left(a_{i j}\right)$, where $a_{i j} \in A$. Let $Z$ be as in the definition of Property P2, so that $Z Z^{*}=s_{11}$. Put $J_{n}(a)=Z_{k} \phi_{n}(a) Z^{*}$ for all $a \in A$. Then $J_{n}$ maps $A$ into $C=s_{11} B s_{11}$. Note that $\phi_{n} \otimes \mathrm{id}: M_{k}(A) \rightarrow M_{k}(C)$ is also full. Set $\mathscr{G}=\left\{\left(a_{i j}\right): a_{i j} \in \mathscr{F} \cup\{0\}\right\}$. By applying Lemma 5.5, we see there is $W \in M_{k}(B)$ with $\|W\| \leq 1$ such that

$$
\left\|\Phi(b)-W^{*}\left(\sum_{k=1}^{m} J_{k} \otimes \operatorname{id} b\right) W\right\|<\frac{\varepsilon}{2 n^{2}} \quad \text { for } b \in \mathscr{G} .
$$

We may also assume that $W^{*} W \leq \operatorname{diag}\left(1_{C}, 0, \ldots, 0\right)$. Choose a positive $d \in A$ such that $0 \leq d \leq 1$ and

$$
\|d a-a\|<\frac{\varepsilon}{2 n^{2}} \quad \text { for all } a \in \mathscr{F} .
$$

Let $\left.v_{i}=0, \ldots, 0, d, 0, \ldots, 0\right)$, where the nonzero entry is in $i$-th place. Let $v_{i}^{\prime}$ be the $n \times n$ matrix whose first row is $v_{i}$ and whose remaining rows are zero. Put $r_{i}=\sum_{n=1}^{m} J_{n} \otimes \operatorname{id}\left(v_{i}^{\prime}\right)$. For any $a \in A$,

$$
\left\|r_{i}^{*}\left(\sum_{n=1}^{m} J_{n}(a) \otimes \operatorname{id}\left(a \otimes s_{11}\right)\right) r_{j}-\sum_{n=1}^{m} J_{n} \otimes \operatorname{id}\left(a \otimes s_{i j}\right)\right\|<\frac{\varepsilon}{2 n^{2}} .
$$

Therefore

$$
\left\|\psi_{i j}(a)-W^{*} r_{i}^{*}\left(\sum_{k=1}^{m} J_{k} \otimes \operatorname{id}\left(a \otimes s_{11}\right)\right) r_{j} W\right\|<\frac{\varepsilon}{n^{2}} \quad \text { for all } a \in \mathscr{F} .
$$

Put $V^{\prime}=\left(v_{1}^{\prime} W, v_{2}^{\prime} W, \ldots, v_{n}^{\prime} W\right)$. We view $V^{\prime}$ as an $n \times n$ matrix whose $i$-th column is the nonzero column $v_{i}^{\prime} W$, for $i=1,2, \ldots, n$. Then

$$
\left\|\psi(a)-V^{\prime *} \sum_{k=1}^{m} J_{k} \otimes \operatorname{id}\left(a \otimes e_{11}\right) V^{\prime}\right\|<\varepsilon \quad \text { for } a \in \mathscr{F} .
$$

Define $V=Z^{*} V^{\prime}$. We have

$$
\left\|\psi(a)-V^{*} \sum_{n=1}^{m} \phi_{n}(a) V\right\|<\varepsilon \quad \text { for } a \in \mathscr{F} .
$$

We also note that $V V^{*} \in \operatorname{Her} \sum_{n=1}^{m} \phi_{n}(A)$. 
Lemma 5.7. Let $A$ be a separable $C^{*}$-algebra and let $B$ be a unital $C^{*}$-algebra having properties $P 1$ and $P 2$. Suppose that $\phi_{n}: A \rightarrow B$ is a sequence of homomorphisms such that the embedding $j_{n}: \phi_{n}(A) \rightarrow B$ is full, where $\left\{\phi_{n}(a): n=1,2, \ldots\right\}$ is an orthogonal set in $B$. Suppose that $\psi: A \rightarrow B$ is amenable and such that $\operatorname{ker} \psi \supset \operatorname{ker} \phi_{n}, n=1,2, \ldots$ For any finite subset $\mathscr{F} \subset A$ and $\varepsilon>0$, there exists a contraction $V \in B$ and an integer $K>0$ such that

$$
\left\|\psi(a)-V^{*}\left(\sum_{i=1}^{K} \phi_{i}(a)\right) V\right\|<\varepsilon \quad \text { for } a \in \mathscr{F} \quad \text { and } \quad V V^{*} \in \operatorname{Her} \sum_{i=1}^{K} \phi_{i}(A) .
$$

Proof. Fix a finite subset $\mathscr{F}_{\text {f }}$ and $\varepsilon>0$. Since $\psi$ is amenable, we may as well assume that $\psi=\alpha \circ \beta$, where $\beta: A \rightarrow M_{n}=M_{n}\left(\mathbb{C} \cdot 1_{C}\right)$ and $\alpha: M_{n} \rightarrow B$ are contractive completely positive linear maps (however, $n$ depends on $\mathscr{F}$ as well as $\varepsilon$ ). Write $M_{n}(C) \subset B$ as in the definition Property P2 (see also Proposition 3.10). Put $\mathscr{G}=\beta(\mathscr{F})$. It is convenient to assume that $\mathscr{F}$ lies in the unit ball of $A$ so $\mathscr{G}$ lies the unit ball of $M_{n}\left(\mathbb{C} \cdot 1_{C}\right)$. Note that $\sigma: M_{n} \rightarrow M_{n}(\mathbb{C}) \subset B$ is full. There exists an integer $m>0$ and a contraction $Z \in M_{m}(B)$ such that

$$
\left\|\alpha(b)-Z^{*}\left(b \operatorname{Id}_{m}\right) Z\right\|<\frac{1}{4} \varepsilon \quad \text { for } b \in \mathscr{G},
$$

where $\operatorname{Id}_{m}$ is the $m \times m$ identity matrix It follows from Lemma 5.6 that there is $N(1)>1$ and a contraction $W_{1} \in B$ such that

$$
\left\|\beta(a)-W_{1}^{*} \sum_{i=1}^{N(1)} \phi_{i}(a) W_{1}\right\|<\frac{\varepsilon}{4 m} \quad \text { for } a \in \mathscr{F}
$$

as well as integers $N(k+1)>N(k)$ and a contractions $W_{k} \in B$ such that

$$
\left\|\beta(a)-W_{k+1}^{*} \sum_{i=N(k)+1}^{N(k+1)} \phi_{i}(a) W_{k+1}\right\|<\frac{\varepsilon}{4 m} \quad \text { for } a \in \mathscr{F} \text { and } k=1,2, \ldots
$$

Note that

$$
\left\|\alpha \circ \beta(a)-Z^{*}\left(\beta(a) \operatorname{Id}_{m}\right) Z\right\|<\frac{1}{2} \varepsilon \quad \text { for } a \in \mathscr{F} .
$$

It follows that

$$
\left.\| \psi(a)-Z^{*}\left(\operatorname{diag}\left(W_{1}^{*} \sum_{i=1}^{m(1)} \phi_{i}(a) W_{1}, \cdots, W_{m} \sum_{i=N(m-1)+1}^{N(m)} \phi_{i}(a)\right) W_{m}\right)\right) Z \|<\frac{1}{2} \varepsilon
$$

for all $a \in \mathscr{F}$. There exist $d_{i} \in \operatorname{Her}\left(\phi_{i}(A)\right)_{+}$with $0 \leq d_{i} \leq 1$ such that

$$
\left\|d_{i} \phi_{i}(a)-\phi_{i}(a)\right\|<\frac{\varepsilon}{2 m} \quad \text { and } \quad\left\|d_{i} \phi_{i}(a) d_{i}-\phi_{i}(a)\right\|<\frac{\varepsilon}{2 m}
$$

for all $a \in \mathscr{F}$. Note that $d_{i} d_{j}=0$ if $i \neq j, i, j=1,2, \ldots, m$. Now let $Y$ be the $n \times n$ matrix whose first row is $\left(d_{1}, d_{2}, \ldots, d_{m}\right)$ and the rest are zero. Put 
$W=\operatorname{diag}\left(W_{1}, W_{2}, \ldots, W_{m}\right)$ and $V=Y W Z$. Then

$$
\left\|\operatorname{diag}\left(W_{1}^{*} \sum_{i=1}^{m(1)} \phi_{i}(a) W_{1}, \ldots, W_{m} \sum_{i=N(m-1)+1}^{N(m)} \phi_{i}(a) W_{m}\right)-W^{*} Y^{*} \sum_{k=1}^{N(m)} \phi_{k}(a) Y W\right\|
$$

is less than $\frac{1}{2} \varepsilon$ for $a \in \mathscr{F}$. Moreover

$$
\left\|\psi(a)-V^{*} \sum_{k=1}^{N(m)} \phi_{k}(a) V\right\|<\varepsilon \quad \text { for } a \in \mathscr{F}_{F} \quad \text { and } \quad V V^{*} \in \operatorname{Her} \sum_{k=1}^{N(m)} \phi_{k}(A) .
$$

\section{Commutants in the ultrapower of corona algebras}

Definition 6.1. Recall that a family $\omega$ of subsets of $\mathbb{N}$ is an ultrafilter if

(i) $X_{1}, \ldots, X_{n} \in \omega$ implies $\bigcap_{i=1}^{n} X_{i} \in \omega$,

(ii) $\varnothing \notin \omega$,

(iii) if $X \in \omega$ and $X \subset Y$, then $Y \in \omega$, and

(iv) if $X \subset \mathbb{N}$ then either $X$ or $\mathbb{N} \backslash X$ is in $\omega$.

An ultrafilter is said to be free if $\bigcap_{X \in \omega} X=\varnothing$. The set of free ultrafilters is identified with elements in $\beta \mathbb{N} \backslash \mathbb{N}$, where $\beta \mathbb{N}$ is the Stone-Çech compactification of $\mathbb{N}$.

A sequence $\left\{x_{n}\right\}$ (in a normed space) is said to converge to $x_{0}$ along $\omega$, written $\lim _{\omega} x_{n}=x_{0}$, if for any $\varepsilon>0$ there exists $X \in \omega$ such that $\left\|x_{n}-x_{0}\right\|<\varepsilon$ for all $n \in X$.

Let $\left\{B_{n}\right\}$ be a sequence of $C^{*}$-algebras. Fix an ultrafilter $\omega$. The ideal of $l^{\infty}\left(\left\{B_{n}\right\}\right)$ consisting of those sequences $\left\{a_{n}\right\}$ in $l^{\infty}\left(\left\{B_{n}\right\}\right)$ such that $\lim _{\omega}\left\|a_{n}\right\|=0$ is denoted by $c_{\omega}\left(\left\{B_{n}\right\}\right)$. Define

$$
q_{\omega}\left(\left\{A_{n}\right\}\right)=l^{\infty}\left(\left\{B_{n}\right\}\right) / c_{\omega}\left(\left\{B_{n}\right\}\right) .
$$

If $B_{n}=B, n=1,2, \ldots$, we write $c_{\omega}(B)$ for $c_{\omega}\left(\left\{B_{n}\right\}\right)$ and $q_{\omega}(A)$ for $q_{\omega}\left(\left\{A_{n}\right\}\right)$.

Lemma 6.2. Let $A$ be a $C^{*}$-algebra, I an ideal of $A$, and let $a \in A \backslash\{0\}$ be such that $0 \leq a \leq 1$. Suppose that $a \notin I$. Then there is $b \in C^{*}(a)$ with $0 \leq b \leq 1$ and $\|b\|=1$ such that if $c \in C^{*}(b) \backslash J$, then $c \notin I$, where

$$
J=\left\{f(b): f \in C_{0}(\operatorname{sp}(b) \backslash\{0\}), f(1)=0\right\} .
$$

Proof. Let $\pi: A \rightarrow A / I$ be the quotient map. Then $\pi(a) \neq 0$. Suppose that $\xi \in \operatorname{sp}(\pi(a)) \backslash\{0\}$. Note $\operatorname{sp}(\pi(a)) \subset \operatorname{sp}(a)$. Let $f \in C_{0}(\operatorname{sp}(a) \backslash\{0\})$ such that $f(\xi)=1$ and $0<f(t)<1$ for all other $t \in \operatorname{sp}(a) \backslash\{0\}$. Set $b=f(a)$. Then, $\pi(b) \neq 0$ and $\|\pi(b)\|=1$. If $c \notin J, c=g(b)$ for some $g \in C_{0}(\operatorname{sp}(b) \backslash\{0\})$ such that $g(1) \neq 0$. Thus $c=g \circ f(a)$. Note that $g \circ f(\xi) \neq 0$. It follows that $\pi(c)=\pi(g \circ f(a)) \neq 0$. Therefore $c \notin I$. 
Lemma 6.3. Let $B$ be a unital $C^{*}$-algebra and let $a \in B$ be an element with $0 \leq a \leq 1$. Suppose that there is $x \in B$ such that $x^{*} a x=1$. Then there exists an element $b \in C^{*}(a)$ such that $c$ is full for all $c \in C^{*}(b) \backslash J$, where

$$
J=\left\{f(b): f \in C_{0}(\operatorname{sp}(b) \backslash\{0\}), f(1)=0\right\} .
$$

Proof. Put $v=a^{1 / 2} x$. Then $v^{*} v=1$ and $v v^{*}=q$ for some projection $q \in B$. Note that $q \in \operatorname{Her}\left(a^{1 / 2} x x^{*} a^{1 / 2}\right) \subset \operatorname{Her}(a)$. For any $0<\varepsilon<\frac{1}{4}$, there is $N>0$ such that

$$
\left\|f_{n}(a) p-p\right\|<\frac{1}{2} \varepsilon \quad \text { for all } n \geq N,
$$

with $f_{n}$ is as in (1-1). It follows that

$$
\left\|f_{n}(a) p f_{n}(a)-p\right\|<\varepsilon \quad \text { for all } n \geq N .
$$

Hence there is a projection $q \in \operatorname{Her}\left(f_{N}(a)\right)$ and a partial isometry $w \in B$ such that $w^{*} q w=1$ and $w w^{*}=q$. Thus $f_{N+1}(a) q=q$. Put $b=f_{N+1}(a)$. For any function $g \in C_{0}((0,1])$, if $g(1) \neq 0$, then $g(b) q=q$. It follows that $w^{*} g(b) w=1$, so $g(b)$ is full and the lemma follows.

Lemma 6.4. Let $A$ be a unital separable $C^{*}$-subalgebra of a unital $C^{*}$-algebra $B$ which has properties $P 1$ and $P 3$. Suppose that every nonzero element in $A$ is full in $B$. Then there exists a sequence of sequences of positive elements $\left\{a_{n}^{(i)}\right\}$, $i=1,2, \ldots$ with $0 \leq a_{n}^{(i)} \leq 1$ satisfying the following:

(1) $\lim _{n \rightarrow \infty}\left\|a_{n}^{(i)} a-a a_{n}^{(i)}\right\|=0$ for all $a \in A$ and $i=1,2, \ldots$

(2) $\lim _{n \rightarrow \infty}\left\|a_{n}^{(i)} a_{n}^{(j)}\right\|=0$ if $i \neq j$.

(3) $\Pi\left(\left\{a_{n}^{(i)}\right\}\right) \Pi \circ J(a)$ is full in $q_{\omega}(A)$ for any free ultrafilter $\omega \in \beta \mathbb{N} \backslash \mathbb{N}$, where $J: B \rightarrow l^{\infty}(B)$ is defined by $J(b)=(b, b, \ldots, b, \ldots)$ for $b \in B$ and $\Pi:$ $l^{\infty}(B) \rightarrow q_{\omega}(B)$ is the quotient map.

Proof. For each nonzero element $0 \leq a \leq 1$ in $A$, define

$$
r(a)=\inf \left\{\|x\|: x^{*} a x=1\right\} .
$$

Let $b_{1}, b_{2}, \ldots, b_{n}, \ldots$ be a dense sequence of the unit ball of $A$. We may assume that $\left\{b_{n}\right\}$ contains a subsequence of positive elements which is dense in the positive part of the unit ball. For each $0 \leq b_{k} \leq 1$ in the sequence, from the assumption, there is $x_{k} \in B$ such that $x_{k}^{*} b_{k} x_{k}=1$ and $\left\|x_{k}\right\| \leq \frac{4}{3} r\left(b_{k}\right)$. Let $D$ be the separable $C^{*}$-subalgebra generated by $A$ and $\left\{x_{k}\right\}$.

We claim that, for each nonzero $a \in A$ with $0 \leq a \leq 1$ there is $x \in D$ such that $x^{*} a x=1$. There is $z \in B$ such that $z^{*} a z=1$ and $\|z\|<\frac{4}{3} r(a)$. There is $b_{k}$ with $0 \leq b_{k} \leq 1$ for which

$$
\left\|a-b_{k}\right\|<\frac{1}{8\left(\frac{4}{3} r(a)+1\right)^{2}} \text {. }
$$


Then

$$
\left\|z^{*} b_{k} z-1\right\| \leq\left\|z^{*}\left(b_{k}-a\right) z\right\| \leq \frac{1}{8} .
$$

We obtain $y \in D$ with $\|y\|<\frac{8}{7}$ such that

$$
y^{*} z^{*} b_{k} z y=1 .
$$

It follows that $r\left(b_{k}\right) \leq \frac{8}{7} r(a)$. Hence there is $x_{k} \in D$ with $\left\|x_{k}\right\| \leq \frac{4}{3} \cdot \frac{8}{7} r(a)$ such that $x_{k}^{*} b_{k} x_{k}=1$. It follows that

$$
\left\|x_{k}^{*} a x_{k}-1\right\| \leq\left\|x_{k}^{*}\left(a-b_{k}\right) x_{k}\right\|<\left(\frac{1}{8}\left(\frac{4}{3} r(a)+1\right)^{2}\right)\left(\frac{4}{3} \cdot \frac{8}{7} r(a)\right)^{2}<\frac{8}{49}<1 .
$$

Thus there is $d \in D$ such that

$$
d^{*} x_{k} a x_{k} d=1 .
$$

This proves the claim.

Now since $B$ has property $\mathrm{P} 3$ and $D$ is separable, there exists a sequence of sequences of nonzero elements $\left\{a_{n}^{(i)}\right\}$ in $B$ with $0 \leq a_{n}^{(i)} \leq 1$ such that

(i) $\lim _{n \rightarrow \infty}\left\|a_{n}^{(i)} d-d a_{n}^{(i)}\right\|=0$ for all $d \in D$,

(ii) $\lim _{n \rightarrow \infty}\left\|a_{n}^{(i)} a_{n}^{(j)}\right\|=0$ if $i \neq j$, and

(iii) for each $i,\left\{a_{n}^{(i)}\right\}$ is full in $l^{\infty}(A)$.

Thus (1) and (2) follow. To see (3), let $a \in A$. From the claim, there is $d \in D$ such that

$$
d^{*} a d=1 .
$$

Put $a_{i}=\left\{a_{n}^{(i)}\right\}$. Then, by Proposition 4.3, there is $z \in l^{\infty}(A)$ such that $z^{*} a_{i} z=1$. Note that (i) implies that

$$
\Pi\left(a_{i}\right) \Pi \circ J(b)=\Pi \circ J(b) \Pi\left(a_{i}\right) \text { for all } b \in D .
$$

Put $g=\Pi \circ J(d) \Pi(z)$. Then

$$
\begin{aligned}
g^{*} \Pi\left(a_{i}\right) \Pi \circ J(a) g & =\Pi\left(z^{*}\right) \Pi \circ J\left(d^{*}\right) \Pi\left(a_{i}\right) \Pi \circ J(a) \Pi \circ J(d) \Pi(z) \\
& =\Pi\left(z^{*}\right) \Pi\left(a_{i}\right) \Pi \circ J\left(d^{*}\right) \pi \circ J(a) \Pi \circ J(d) \Pi(z) \\
& =\pi\left(z^{*}\right) \Pi\left(a_{i}\right) \Pi(z)=1 .
\end{aligned}
$$

Lemma 6.5. Let $A$ be a unital separable amenable $C^{*}$-algebra and $B$ a unital $C^{*}$-algebra having properties $P 1, P 2$ and $P 3$. Let $\omega \in \beta \mathbb{N} \backslash \mathbb{N}$ be a free ultrafilter. Suppose that $\tau: A \rightarrow B$ is a full unital embedding. Let $\tau_{\infty}: A \rightarrow l^{\infty}(B)$ be defined by $\tau_{\infty}(a)=(\tau(a), \tau(a), \ldots)$ and let $\psi=\Pi \circ \tau_{\infty}$, where $\Pi: l^{\infty}(B) \rightarrow q_{\omega}(B)$. Then there is a unital $C^{*}$-subalgebra $C \cong 0_{\infty}$ in the commutant of $\psi(A)$ in $q_{\omega}(B)$. 
Proof. Let $\left\{a_{n}^{(i)}\right\}$ be the sequence of sequences of elements given by Lemma 6.4. Put $a_{i}=\left\{a_{n}^{(i)}\right\}, i=1,2, \ldots$

Applying Lemma 6.3, and introducing and using $D$ as in the proof of Lemma 6.4, we may assume that each $a_{i}$ has the property that $\operatorname{sp}\left(a_{i}\right) \subset[0,1]$ and $f\left(\Pi\left(a_{i}\right)\right)$ is full for all $0 \leq f \leq 1$ in $C_{0}((0,1])$ for which $f(1) \neq 0$.

Let $X=(0,1]$ and fix $i$. Define $\phi_{j}^{\prime}, L^{\prime}: C_{0}(X) \otimes A \rightarrow q_{\omega}(B)$ by $\phi_{i}^{\prime}(f \otimes a)=$ $f\left(\Pi\left(a_{i}\right)\right) \psi(a)$ and $L^{\prime}(f \otimes a)=f(1) \psi(a)$ for $a \in A$. By Lemma 6.4(3), $\phi_{i}^{\prime}$ is full. Let $\left\{\mathscr{F}_{j}\right\}$ be an increasing sequence of finite subsets of $A$ for which $\bigcup_{n=1}^{\infty} \mathscr{F}_{j}$ is dense in $A$ and let $\left\{g_{n}\right\}$ be a dense sequence of $C_{0}((0,1])$.

Let $\left\{a_{i(k)}\right\}_{k=1}^{\infty}$ be a subsequence of $\left\{a_{i}\right\}$. It follows from Lemma 5.7 that there exists $s_{n} \in B$ such that

$$
\left\|s_{n}^{*}\left(\sum_{k=1}^{m(n)} g_{j}\left(a_{i(k)}\right) \psi(a)\right) s_{n}-g_{j}(1) \psi(a)\right\|<\frac{1}{2^{n}} \quad \text { for } a \in \mathscr{F}_{n} \text { and } j=1,2, \ldots, n .
$$

Moreover, $s_{n} s_{n}^{*} \in \operatorname{Her}\left(\sum_{k=1}^{m(n)}\left(a_{i(k)}\right) \psi(A)\right)$. Suppose that $s_{n}=\Pi\left(\left(s_{n, 1}, s_{n, 2}, \ldots\right)\right)$, $n=1,2, \ldots$. We may assume that

$$
\left\|s_{n, k(n)}^{*}\left(\sum_{k=1}^{m(n)} g_{j}\left(a_{k(n)}^{i(k)}\right) \tau(a)\right) s_{n, k(n)}-g_{j}(1) \tau(a)\right\|<\frac{1}{2^{n}} \quad \text { for } n=1,2, \ldots
$$

Now put $t_{n}=s_{n, k(n)}, t^{\prime}=\left(t_{1}, t_{2}, \ldots\right)$ and $t=\Pi\left(t^{\prime}\right)$. Define $\Phi: C_{0}(X) \otimes A \rightarrow l^{\infty}(B)$ by

$$
\Phi(f \otimes a)=\left\{\sum_{k=1}^{m(n)} f\left(a_{k(n)}^{(i(k)}\right) \tau(a)\right\} \quad \text { for all } f \in C_{0}(X) \text { and } a \in A .
$$

It follows that

$$
t^{*} \Pi \circ \Phi(f \otimes a) t=f(1) \psi(a) \text { for all } f \in C_{0}(X) \text { and } a \in A .
$$

Put $b(\{i(k)\})=\Pi\left(\left\{a_{k(n)}^{i(k)}\right\}\right)$. Note that $0 \leq b(\{i(k)\}) \leq 1$. We have $($ with $\iota(t)=t$ for all $t \in(0,1])$

Put

$$
t^{*} b(\{i(k)\}) t=\iota(1)=1_{q_{\omega(B)}} .
$$

$$
w(\{i(k)\})=b(\{i(k)\})^{1 / 2} t \quad \text { and } \quad q=b(\{i(k)\})^{1 / 2} t t^{*} b\left(\{(i(k)\})^{1 / 2} .\right.
$$

Since $b(\{i(k)\}) \in \psi(A)^{\prime}$ and $\iota(1)=1$, we have

$$
\begin{aligned}
t^{*} b(\{i(k)\})^{1 / 2} \psi(a) b(\{i(k)\})^{1 / 2} t & =t^{*} b(\{i(k)\}) \psi(a) t=\iota(1) \psi(a) \\
& =\psi(a) \quad \text { for all } a \in A .
\end{aligned}
$$

It follows from [Rørdam 2002, 6.36] that $w(\{i(k)\})=b(\{i(k)\})^{1 / 2} t \in \psi(A)^{\prime}$. If $\{i(k)\}$ and $\left\{i(k)^{\prime}\right\}$ are disjoint infinite subsets of $\mathbb{N}$, the corresponding projections $q$ and $q^{\prime}$ are orthogonal. Thus there is a sequence of isometries $v_{k} \in \psi(A)^{\prime}$ such 
that $v_{k}^{*} v_{k}=1_{q_{\omega}(B)}$ and $1 \geq \sum_{k=1}^{n} v_{k} v_{k}^{*}, n=1,2, \ldots$ Thus $\psi(A)^{\prime}$ admits a unital embedding of $\mathrm{O}_{\infty}$,

\section{Full extensions}

Definition 7.1. Let $\operatorname{Ext}(A, B)$ be the set of stable unitary equivalence classes of extensions of the form (2-1). When $A$ is amenable, it is known (by work of Arveson, Choi and Effros) that $\operatorname{Ext}(A, B)$ is a group. Moreover, the group can be identified with $K K^{1}(A, B)$. Let $\mathscr{T}(A, B)$ be the set of all stable unitary equivalence classes of approximately trivial extensions. It is known that $\mathscr{T}(A, B)$ is a subgroup of $K K^{1}(A, B)$ (see [Lin 2005a]). Following Rørdam, one defines $K L^{1}(A, B)=$ $K K^{1}(A, B) / \mathscr{T}(A, B)$.

Let $G_{1}, G_{2}, G_{3}$ be abelian groups. A group extension $0 \rightarrow G_{1} \rightarrow G_{3} \rightarrow$ $G_{2} \rightarrow 0$ is said to be pure if every finitely generated subgroup of $G_{2}$ lifts. Denote by $\operatorname{Pext}\left(G_{2}, G_{1}\right)$ the set of all pure extensions and by $E\left(G_{2}, G_{1}\right)$ the quotient $\operatorname{ext}_{\mathbb{Z}}\left(G_{2}, G_{1}\right) / \operatorname{Pext}\left(G_{2}, G_{1}\right)$.

If $A$ satisfies the Approximate Universal Coefficient Theorem, or AUCT (see [Lin 2005a]), one has the following short exact sequence:

$$
0 \rightarrow E\left(K_{i}(A), K_{i}(B)\right) \rightarrow K L^{1}(A, B) \rightarrow \operatorname{Hom}\left(K_{i}(A), K_{i-1}(B)\right) \rightarrow 0 .
$$

So $K L^{1}(A, B)$ may be computable in theory. It should be noted every separable amenable $C^{*}$-algebra which satisfies the Universal Coefficient Theorem (UCT) satisfies the AUCT. Rosenberg and Schochet [1987] have shown that every separable $C^{*}$-algebras in the so-called bootstrap class satisfies the UCT (therefore the AUCT). We also use the notation $K L(A, B)=K L^{1}(A, S B)$.

As mentioned in the introduction, two stably unitarily equivalent extensions are in general not unitarily equivalent and trivial extensions are not unitarily equivalent. Furthermore, an essential extension which is zero in $K K^{1}(A, B)$ may not be trivial (or approximately trivial). We will use $K L^{1}(A, M(B) / B$ ) to give a classification of full essential extensions up to approximately unitary equivalence.

Proposition 7.2. Let $D$ be a unital $C^{*}$-algebra for which there is a unital embedding from $\mathrm{O}_{2}$ to $D$. Let $h_{1}, h_{2}: \mathrm{O}_{2} \rightarrow D$ be two full homomorphisms. Suppose that $h_{1}\left(1_{\mathrm{O}_{2}}\right) \sim h_{2}\left(1_{\mathrm{O}_{2}}\right)$. Then there is a sequence of partial isometries $v_{n}$ such that

$$
v_{n}^{*} v_{n}=h_{2}\left(1_{\mathbb{O}_{2}}\right), v_{n} v_{n}^{*}=h_{1}\left(1_{\mathfrak{O}_{2}}\right) \quad \text { and } \lim _{n \rightarrow \infty}\left\|v_{n}^{*} h_{1}(a) v_{n}-h_{2}(a)\right\|=0
$$

for all $a \in \mathrm{O}_{2}$.

Proof. This is the combination of Theorem 6.5 and Lemma 7.2 in [Lin 2007].

Lemma 7.3. Let $A$ be a unital separable $C^{*}$-algebra and let $B$ and $C$ be unital $C^{*}$ algebras such that $B \otimes \mathrm{O}_{2}$ is a unital $C^{*}$-subalgebra of $C$ and $C$ has property $P 1$. 
Suppose that $h_{1}, h_{2}: A \rightarrow B \otimes \mathbb{C} \cdot 1 \subset B \otimes O_{2}$ are two unital full monomorphisms. Then $h_{1}$ and $h_{2}$ are approximately unitarily equivalent in $C$.

Proof. By [Rørdam 1994] we have $\mathrm{O}_{2} \cong \mathrm{O}_{2} \otimes \mathrm{O}_{2}$. Let $p_{n}=1_{B} \otimes q_{n} \otimes 1_{\mathfrak{O}_{2}}$, where $\left\{q_{n}\right\}$ is a sequence of mutually orthogonal nonzero projections in $\mathrm{O}_{2}$. Note that $p_{n} \sim 1_{B \otimes \mathbb{Q}_{2} \otimes \mathbb{O}_{2}}, n=1,2, \ldots$ Define $\phi_{i}(a)=p_{i} h_{1}(a)$ and $\psi_{i}(a)=p_{i} h_{2}(a)$ for all $a \in A$. Also define $\Phi_{n}(a)=\left(1-\sum_{i=1}^{n} p_{i}\right) h_{1}(a)$ and $\Psi_{n}(a)=\left(1-\sum_{i=1}^{n} p_{i}\right) h_{2}(a)$ for all $a \in A$. Then, for each $n, h_{1}=\sum_{i=1}^{n} \phi_{i} \oplus \Phi_{n}$ and $h_{2}=\sum_{i=1}^{n} \psi_{i} \oplus \Psi_{n}$. Note that $\phi_{i}, \Phi_{n}, \psi_{i}$ and $\Psi_{n}$ are all full. Now we work in $B \otimes \mathcal{O}_{2} \otimes 1$. There are partial isometries $v_{i, j} \in \mathrm{O}_{2}$ such that $v_{i, j}^{*} v_{i, j}=p_{j}$ and $v_{i, j} v_{i, j}^{*}=p_{i}$ for $i, j=1,2, \ldots, n$ and

$$
v_{n+1, j}^{*} v_{n+1, j}=p_{j}, \quad v_{n+1, j} v_{n+1, j}^{*}=1-\sum_{i=1}^{n} p_{i} \quad \text { for } j=1,2, \ldots, n \text {. }
$$

Put $w_{i, j}=1 \otimes v_{i, j} \otimes 1$. Then we also have

$$
w_{i, 1}^{*} \phi_{1} w_{i, 1}=\phi_{i} \quad \text { for } i=1,2, \ldots, n \quad \text { and } \quad w_{n+1,1}^{*} \phi_{1} w_{n+1,1}=\Phi_{n} .
$$

Let $\mathscr{F}_{1}, \mathscr{F}_{2}, \ldots, \mathscr{F}_{n}, \ldots$ be an increasing sequence of finite subsets of $A$ such that $\bigcup_{n=1}^{\infty} \mathscr{F}_{n}$ is dense in $A$. It follows from [Lin 2001, Lemma 5.4.2] that, for each $n$, there are isometries $u_{n}, v_{n} \in B \otimes \mathrm{O}_{2} \otimes 1$ such that

$$
\left\|u_{n}^{*} h_{1}(a) u_{n}-h_{2}(a)\right\|<1 / n \quad \text { and } \quad\left\|v_{n}^{*} h_{2}(a) v_{n}-h_{1}(a)\right\|<1 / n \quad \text { for } a \in \mathscr{F}_{n} .
$$

Note that the relative commutant of $B \otimes \mathrm{O}_{2} \otimes 1$ contains a unital $C^{*}$-subalgebra $1_{B} \otimes 1_{O_{2}} \otimes \mathrm{O}_{2}$ which is isomorphic to $\mathrm{O}_{2}$. It follows from [Kirchberg and Phillips $2000,1.10]$ that $h_{1}$ and $h_{2}$ are approximately unitarily equivalent.

Lemma 7.4. Let $A$ be a unital separable nuclear $C^{*}$-algebra, let $B_{1}$ and $B_{2}$ be two unital $C^{*}$-algebra and let $C$ be another unital $C^{*}$-algebra. Suppose that $j_{i}$ : $B_{i} \otimes \mathrm{O}_{2} \rightarrow C$ are two full monomorphisms so that $j_{1}(1) \sim j_{2}(1)$ and $h_{i}: A \rightarrow B_{i}$ are two full unital monomorphisms. Then there is a sequence of partial isometries $v_{n} \in C$ such that $v_{n}^{*} v_{n}=j_{1}(1), v_{n} v_{n}^{*}=j_{2}(1)$ and

$$
\lim _{n \rightarrow \infty}\left\|v_{n}^{*}\left(j_{2} \circ h_{2}(a)\right) v_{n}-j_{1} \circ h_{1}(a)\right\|=0 \quad \text { for all } a \in A .
$$

Proof. To simplify notation, we may assume that $j_{1}(1)=j_{2}(1)$. Therefore we may assume that both $j_{1}$ and $j_{2}$ are unital. Define $J_{i}: B_{i} \otimes \mathrm{O}_{2} \rightarrow l^{\infty}(C)$ by $J_{i}(b)=\left(j_{i}(b), j_{i}(b), \ldots\right)$ for $b \in B_{i} \otimes O_{2}$ and $H_{i}=J_{i} \circ h_{i}$, respectively, $i=1,2$. Note that these maps are full in $l^{\infty}(C)$. Since there is a unital $\mathrm{O}_{2}$ embedding to $l^{\infty}(C)$, by Proposition 7.2, we obtain unitaries $u_{n} \in C$ such that

$$
\lim _{n \rightarrow \infty}\left\|u_{n}^{*} J_{2}(1 \otimes b) u_{n}-J_{1}(1 \otimes b)\right\|=0 \quad \text { for all } b \in \mathcal{O}_{2} .
$$

Denote $U=\left\{u_{n}\right\}$ in $l^{\infty}(C)$. Let $\omega$ be a free ultrafilter on $\mathbb{N}$ and $\pi: l^{\infty}(C) \rightarrow q_{\omega}(C)$ be the quotient map. Let $D$ be the $C^{*}$-subalgebra generated by $\pi \circ J_{1}\left(B_{1} \otimes \mathbb{C} \cdot 1_{\mathscr{C}_{2}}\right)$ 
and $\pi \circ$ ad $U \circ J_{2}\left(B_{2} \otimes \mathbb{C} \cdot 1_{\bigcirc_{2}}\right)$. It follows that $D^{\prime}$, the commutant of $D$, contains $J_{1}\left(1_{B_{1}} \otimes \mathrm{O}_{2}\right)$ which is isomorphic to $\mathrm{O}_{2}$. Therefore we may write $D \subset D \otimes \mathrm{O}_{2}$. Now $\pi \circ H_{1}$ and $\pi \circ$ ad $W \circ H_{2}$ are two full unital monomorphisms from $A$ into $D \subset D \otimes \mathrm{O}_{2}$. It follows from Lemma 7.3 that $\pi \circ H_{1}$ and $\pi \circ$ ad $W \circ H_{2}$ are approximately unitarily equivalent. It follows from [Rørdam 2002, Lemma 6.2.5] that $j_{1} \circ h_{1}$ and $j_{2} \circ h_{2}$ are approximately unitarily equivalent.

Theorem 7.5. Let $A$ be a unital separable amenable $C^{*}$-algebra and let $B$ be a unital $C^{*}$-algebra which has properties $P 1, P 2$ and $P 3$. Let $j_{o}: A \rightarrow \mathrm{O}_{2} \rightarrow B$ be a full embedding of $A$ into $B$ which factors through $\mathrm{O}_{2}$. Suppose that $\tau: A \rightarrow B$ is a full monomorphism. Then there is a sequence of partial isometries $V_{n} \in M_{2}(B)$ such that $V_{n}^{*} V=1_{B} \oplus j_{o}\left(1_{A}\right), V_{n} V_{n}^{*}=1_{B}$ and

$$
\lim _{n \rightarrow \infty}\left\|V_{n}\left(\tau \oplus j_{o}\right)(a) V_{n}^{*}-\tau(a)\right\|=0 \quad \text { for all } a \in A .
$$

Proof. Let $J: B \rightarrow l^{\infty}(B)$ be defined by $J(c)=(c, c, \ldots)$ for $c \in B$. Define $\tau_{\infty}=J \circ \tau$ and $J_{o}=J \circ j_{o}$. Let $\omega$ be a free ultrafilter on $\mathbb{N}$ and $\pi: l^{\infty}(B) \rightarrow q_{\omega}(B)$ be the quotient map. It follows from Lemma 6.5 that $\pi \circ \tau_{\infty}(A)^{\prime}$ contains a unital $C^{*}$-subalgebra which is isomorphic to $\mathrm{O}_{\infty}$. Denote this $C^{*}$-subalgebra by $\mathrm{O}_{\infty}$. Let $q \in \mathrm{O}_{\infty}$ be a nonzero projection such that $[q]=0$ in $K_{0}\left(\mathrm{O}_{\infty}\right)$. There is a $C^{*}$ subalgebra $C$ of $\mathrm{O}_{\infty}$ for which $1_{C}=q$ and $C \cong \mathrm{O}_{2}$. Put $\tau_{0}(a)=q \pi \circ \tau_{\infty}(a)$. So we may view $\tau_{0}$ is a unital full homomorphism from $A$ into $\tau_{0}(A) \otimes \mathrm{O}_{2}$. Since $\mathrm{O}_{2} \cong \mathbb{O}_{2} \otimes \mathbb{O}_{2}$ by [Rørdam 1994], it follows from Lemma 7.4 that $\tau_{0} \oplus \pi \circ J_{o}$ and $\tau_{0}$ are approximately unitarily equivalent. Thus $\pi \circ \tau_{\infty}$ and $\pi \circ \tau_{\infty} \oplus \pi \circ J_{o}$ are approximately unitarily equivalent. It follows from [Rørdam 2002, 6.2.5] that $\tau$ and $\tau \oplus j_{o}$ are approximately unitarily equivalent.

Proof of Theorem 2.6. Since $A$ is separable, there is a unital embedding $j: A \rightarrow \mathrm{O}_{2}$, by [Kirchberg and Phillips 2000, 2.8]. Since $B$ has property P2, there is a full monomorphism $\sigma: \mathrm{O}_{2} \rightarrow B$. Define $\bar{j}=\sigma \circ j$. Note $\bar{j}$ is full. Let $\varepsilon>0$ and $\mathscr{F} \subset A$ be a finite subset. It follows from [Lin 2005a, Theorem 3.9] that there is an integer $n$ and a unitary $v \in M_{n+1}(B)$ such that

$\left\|v^{*} \operatorname{diag}\left(h_{1}(a), \bar{j}(a), \bar{j}(a), \ldots, \bar{j}(a)\right) v-\operatorname{diag}\left(h_{2}(a), \bar{j}(a), \bar{j}(a), \ldots, \bar{j}(a)\right)\right\|<\frac{1}{4} \varepsilon$

for all $a \in \mathscr{F}$. On the other hand, by Proposition 7.2, there is an isometry $u \in$ $M_{n}\left(\pi \circ \sigma\left(O_{2}\right)\right)$ with $u u^{*}=1_{\pi \circ \sigma\left(O_{2}\right)}$ such that

$$
\left\|u^{*} \bar{j}(a) u-\operatorname{diag}(\bar{j}(a), \bar{j}(a), \ldots, \bar{j}(a))\right\|<\frac{1}{4} \varepsilon
$$

for $a \in \mathscr{F}$. Thus, we obtain an isometry $w \in M_{2}(B)$ with $w w^{*}=1_{B}$ such that

$$
\left\|w^{*} \operatorname{diag}\left(h_{1}(a), \bar{j}(a)\right) w-\operatorname{diag}\left(h_{2}, \bar{j}(a)\right)\right\|<\frac{1}{2} \varepsilon \quad \text { for all } a \in \mathscr{F} .
$$


By applying Theorem 7.5, we obtain a partial isometry $z \in B$ such that $z^{*} h_{1}\left(1_{A}\right) z=$ $h_{2}\left(1_{A}\right), z h_{2}\left(1_{A}\right) z^{*}=h_{1}\left(1_{A}\right)$ and

$$
\left\|z^{*} h_{1}(a) z-h_{2}(a)\right\|<\varepsilon \quad \text { for all } a \in \mathscr{F} .
$$

Remark 7.6. If both $h_{1}$ and $h_{2}$ are unital, it is clear that $z$ can be chosen to be unitary. If one of them is unital and the other is not, $z$ can never be unitary. Suppose that both are not unital. Since $B$ has properties P1, P2 and P3, we obtain full $\mathrm{O}_{2}$ embeddings into $h_{1}\left(1_{A}\right) B h_{1}\left(1_{A}\right)$ and $h_{2}\left(1_{A}\right) B h_{2}\left(1_{A}\right)$. Therefore there is a projection $e \leq h_{1}\left(1_{A}\right)$ such that $h_{1}\left(1_{A}\right)$ is equivalent to $h_{1}\left(1_{A}\right)-e$ and $e$ is a full projection. So there is a partial isometry $v \in B$ such that $v^{*} v=h_{1}\left(1_{A}\right)$ and $v v^{*}=h_{1}\left(1_{A}\right)-e$. Thus $1-\operatorname{ad} v^{*} \circ h_{1}\left(1_{A}\right)$ is full. Similarly, there is a partial isometry $w \in B$ with $w^{*} w=h_{2}\left(1_{A}\right)$ such that $1-\operatorname{ad} w^{*} \circ h_{2}\left(1_{A}\right)$ is full. Now apply Theorem 2.6 to the case that $A=\mathbb{C}$. we know that $1-\operatorname{ad} v^{*} \circ h_{1}\left(1_{A}\right)$ and $1-\operatorname{ad} w^{*} \circ h_{2}\left(1_{A}\right)$ are equivalent. This implies that we can choose $z$ to be unitary in the proof of Theorem 2.6 in the case that both $h_{1}$ and $h_{2}$ are not unital.

Corollary 7.7. Theorem 2.6 also holds for the case that $B=q_{\infty}\left(\left\{C_{n}\right\}\right)$, where each $C_{n}$ is a unital purely infinite simple $C^{*}$-algebras.

Proof. It is clear that $B$ has properties P1 and P2. From the proof of Theorem 2.6 above, we only need an absorbing Theorem 7.5 for this $B$. Let $\tau: A \rightarrow B$ be a full monomorphism and $j_{0}: A \rightarrow \mathrm{O}_{2} \rightarrow B$ be a full embedding of $A$ into $B$ which factors through $\mathrm{O}_{2}$. So we may write $j_{0}=\Phi \circ j$, where $j: A \rightarrow \mathrm{O}_{2}$ is a monomorphism and $\Phi: \mathrm{O}_{2} \rightarrow B$ is a full homomorphism. Let $L: A \rightarrow$ $l^{\infty}\left(\left\{C_{n}\right\}\right.$ be a contractive completely positive linear map for which $\pi \circ L=\tau$, where $\pi: l^{\infty}\left(\left\{C_{n}\right\}\right) \rightarrow q_{\infty}\left(\left\{C_{n}\right\}\right)$ is the quotient map. Write $L=\left\{L_{n}\right\}$, where $L_{n}: A \rightarrow C_{n}$ is a contractive completely positive linear map. Let $\phi_{n}: \mathrm{O}_{2} \rightarrow C$ such that $\pi \circ\left\{\psi_{n}\right\}=\Phi$. Denote by $D_{n}$ the separable unital purely infinite simple $C^{*}$ algebra containing $L_{n}(A)$ and $\psi_{n}\left(O_{2}\right)$. Then $q_{\infty}\left(\left\{D_{n}\right\}\right) \subset B$ and $\tau: A \rightarrow q_{\infty}\left(\left\{C_{n}\right\}\right)$ and $j_{0}: A \rightarrow \mathrm{O}_{2} \rightarrow q_{\infty}\left(\left\{C_{n}\right\}\right)$. Thus one can apply [Lin 2004a, 7.5].

Proof of Proposition 2.9. Let $h_{1}: A \rightarrow B \otimes \mathscr{K}$ be a homomorphism. It follows from [Lin 2004a, 4.5] that there is a sequence of asymptotically multiplicative contractive completely positive linear maps $\left\{\phi_{n}\right\}$ from $A$ to $B \otimes \mathscr{K}$ and a sequence of unitaries $u_{n} \in \widetilde{B \otimes \mathscr{K}}$ such that

$$
\lim _{n \rightarrow \infty}\left\|\left(h \oplus \phi_{n}\right)(a)-\operatorname{ad} u_{n} \circ j(a)\right\|=0 \quad \text { for all } a \in A .
$$

Since $B$ has property $\mathrm{P} 2$, it is easy to see that we may assume that $\phi_{n}$ maps $A$ into $B$ and $u_{n}$ are unitaries in $B$. It follows from 6.5 in [Lin 2007] that, for each $k$, there exists a sequence of unitaries $v_{n}(k) \in M_{2}(B)$ such that

$$
\lim _{n \rightarrow \infty}\left\|v_{n}(k)^{*}\left(\phi_{n}(a) \oplus j_{o}(a)\right) v_{n}(k)-\left(\phi_{n+k}(a) \oplus j_{o}(a)\right)\right\|=0 \quad \text { for all } a \in A .
$$


It follows from [Lin 2004a, 4.7] that there exists a homomorphism $h_{1}: A \rightarrow M_{2}(B)$ and a sequence of unitaries $w_{n} \in M_{2}(B)$ such that

$$
\lim _{n \rightarrow \infty}\left\|\operatorname{ad} w_{n} \circ h_{1}(a)-\left(\phi_{n}(a) \oplus j_{o}(a)\right)\right\|=0 \quad \text { for all } a \in A .
$$

By applying the fact that $B$ has property $\mathrm{P} 2$ and applying Proposition 7.2, we obtain a sequence of isometries $z_{n} \in M_{3}(B)$ with $z_{n} z_{n}^{*}=j_{o}\left(1_{A}\right)$ such that

$$
\lim _{n \rightarrow \infty}\left\|\left(h \oplus h_{1} \oplus j_{o}\right)(a)-z_{n}^{*} j_{o}(a) z_{n}\right\|=0 \quad \text { for all } a \in A .
$$

Hence $\left[h_{1}\right]=-[h]$ in $H(A, B)$.

Proof of Corollary 2.10. Combine Proposition 2.9 and Theorem 7.5.

\section{Classification of full extensions}

Definition 8.1. Let $C_{n}$ be a commutative $C^{*}$-algebra with $K_{0}\left(C_{n}\right)=\mathbb{Z} / n \mathbb{Z}$ and $K_{1}\left(C_{n}\right)=0$. Suppose that $A$ is a $C^{*}$-algebra. Put $K_{i}(A, \mathbb{Z} / k \mathbb{Z})=K_{i}\left(A \otimes C_{k}\right)$. One has the following six-term exact sequence (see [Schochet 1984]):

$$
\begin{array}{ccc}
K_{0}(A) & \rightarrow K_{0}(A, \mathbb{Z} / k \mathbb{Z}) \rightarrow K_{1}(A) \\
\uparrow_{\mathbf{k}} & \downarrow_{\mathbf{k}} \\
K_{0}(A) \leftarrow K_{1}(A, \mathbb{Z} / k \mathbb{Z}) \leftarrow K_{1}(A) .
\end{array}
$$

In [Dadarlat and Loring 1996], $K_{i}(A, \mathbb{Z} / n \mathbb{Z})$ is identified with $K K^{i}\left(\mathbb{q}_{n}, A\right)$ for $i=0,1$. As in that paper, we use the notation

$$
\underline{K}(A)=\bigoplus_{\substack{i=0,1 \\ n \in \mathbb{Z}_{+}}} K_{i}(A ; \mathbb{Z} / n \mathbb{Z}) .
$$

By $\operatorname{Hom}_{\Lambda}(\underline{K}(A), \underline{K}(B))$ we mean all homomorphisms from $\underline{K}(A)$ to $\underline{K}(B)$ which respect to direct sum decomposition and the so-called Bockstein operations; see [Dadarlat and Loring 1996]. It follows from these authors' definition that if $x \in$ $K K(A, B)$, the Kasparov product $K K^{i}\left(\mathbb{\square}_{n}, A\right) \times x$ gives an element in $K K^{i}\left(\mathbb{\square}_{n}, B\right)$, which we identify with $\operatorname{Hom}\left(K_{i}(A, \mathbb{Z} / n \mathbb{Z}), K_{0}(B, \mathbb{Z} / n \mathbb{Z})\right)$. Thus one obtains a map $\Gamma: K K(A, B) \rightarrow \operatorname{Hom}_{\Lambda}(\underline{K}(A), \underline{K}(B))$. It was shown in the same paper that if $A$ is in $\mathcal{N}$ then, for any $\sigma$-unital $C^{*}$-algebra $B$, the map $\Gamma$ is surjective and $\operatorname{ker} \Gamma=\operatorname{Pext}\left(K_{*}(A), K_{*}(B)\right)$. In particular,

$$
\Gamma: K L(A, B) \rightarrow \operatorname{Hom}_{\Lambda}(\underline{K}(A), \underline{K}(B))
$$

is an isomorphism. It is shown in [Lin 2005a] that if $A$ satisfies the AUCT, then $\Gamma$ is also an isomorphism from $K L(A, B)$ onto $\operatorname{Hom}_{\Lambda}(\underline{K}(A), \underline{K}(B))$.

Lemma 8.2. Let $B$ be a unital $C^{*}$-algebra which admits a full $\mathrm{O}_{2}$ embedding and let $G_{i}$ be a countable subgroup of $K_{i}(B)(i=0,1)$. There exists a unital separable 
$C^{*}$-algebra $B_{0} \subset B$ which has a full $\mathrm{O}_{2}$ embedding such that $K_{i}\left(B_{0}\right) \supset G_{i}$ and $j_{* i}=\operatorname{id}_{K_{i}\left(B_{0}\right)}$, where $j: B_{0} \rightarrow B$ is the embedding.

Proof. Let $p_{1}, \ldots, p_{n}, \ldots$ be projections and $u_{1}, u_{2}, \ldots, u_{n}, \ldots$ be unitaries in $\bigcup_{k=1}^{\infty} M_{k}(B)$ such that $\left\{p_{n}\right\}$ and $\left\{u_{n}\right\}$ generates of $G_{0}$ and $G_{1}$, respectively. There is a countable set $S$ such that

$$
p_{n}, u_{n} \in \bigcup_{n=1}^{\infty}\left\{\left(a_{i j}\right)_{n \times n} \in M_{n}(B): a_{i j} \in S\right\} .
$$

Let $j_{o}: \mathrm{O}_{2} \rightarrow B$ be a full embedding. Let $p=j\left(1_{\mathscr{O}_{2}}\right)$ and $x_{1}, x_{2}, \ldots, x_{m} \in B$ such that $\sum_{i=1}^{m} x_{i}^{*} p x_{i}=1$. Let $B_{1}$ be the unital separable $C^{*}$-subalgebra generated by $S,\left\{x_{1}, x_{2}, \ldots, x_{m}\right\}$ and $j\left(\mathbb{O}_{2}\right)$. Then $B_{1}$ has a full $O_{2}$ embedding and $p_{n}, u_{n} \in$ $\bigcup_{k=1}^{\infty} M_{k}\left(B_{1}\right)$ for all $n$. Note that $K_{i}\left(B_{1}\right)$ is countable. The embedding $j_{1}: B_{1} \rightarrow B$ gives homomorphisms $\left(j_{1}\right)_{* i}: K_{i}\left(B_{1}\right) \rightarrow K_{i}(B)$. Let $F_{1, i}$ be the subgroup of $K_{0}\left(B_{1}\right)$ generated by $\left\{p_{n}\right\}$ and $\left\{u_{n}\right\}$, respectively. It is clear that $\left(j_{1}\right)_{* i}$ is injective on $F_{1, i}, i=0,1$. In particular, the image of $\left(j_{1}\right)_{* i}$ contains $G_{i}, i=0,1$. Let $N_{1, i}^{\prime}=\operatorname{ker}\left(j_{1}\right)_{* i}$ and let $N_{1, i}$ be the set of all projections (if $i=0$ ), or unitaries (if $i=1)$ in $\bigcup_{k=1}^{\infty} M_{k}\left(B_{1}\right)$ which have images in $N_{1, i}^{\prime}$. Let $\left\{p_{1, n}\right\}$ be a dense subset of projections in $\bigcup_{k=1}^{\infty} M_{k}\left(B_{1}\right)$. There are countable pairs of projections $\left\{e_{n}, e_{n}^{\prime}\right\}$ in $\left\{p_{1, n}\right\}$ such that $\left[e_{n}\right]=\left[e_{n}^{\prime}\right]$ in $K_{0}(B)$. There are $w_{n} \in \bigcup_{k=1}^{\infty} M_{k}(B)$ such that $w_{n}^{*} w_{n}=e_{n} \oplus 1_{k(n)}$ and $w_{n} w_{n}^{*}=e_{n}^{\prime} \oplus 1_{k(n)}$.

Let $\left\{u_{1, n}\right\}$ be a dense subset of unitaries in $\bigcup_{k=1}^{\infty} M_{k}\left(B_{1}\right)$. For each $u_{1, n}$, there are unitaries $z_{1, n, k} \in \bigcup_{j=1}^{\infty} M_{j}(B), k=1,2, \ldots, m(n)$ such that

$$
\left\|z_{1, n, 1}-1\right\|<\frac{1}{2},\left\|z_{1, n, m(n)}-u_{1, n}\right\|<\frac{1}{2} \quad \text { and } \quad\left\|z_{1, n, k}-z_{1, n, k+1}\right\|<\frac{1}{2},
$$

$k=1,2, \ldots, m(n), n=1,2, \ldots$ Let $B_{2}$ be a separable unital $C^{*}$-algebra containing $B_{1}$ such that $\bigcup_{k=1}^{\infty} M_{k}\left(B_{2}\right)$ contains all $\left\{w_{1, n}\right\}$ and $\left\{z_{1, n, k}\right\}$. Note that there is a full embedding of $\mathrm{O}_{2}$ to $B_{2}$. Note also that if $p, q \in \bigcup_{k=1}^{\infty} M_{k}\left(B_{1}\right)$ are projections such that $[p]-[q] \in N_{1,0}$ then $[p]-[q]=0$ in $K_{0}\left(B_{2}\right)$. Similarly, if $u \in B_{1}$ and $[u] \in N_{1,1}$, then $[u]=0$ in $B_{2}$. Suppose that $B_{l}$ has been constructed. Let $j_{l}: B_{l} \rightarrow B$ be the embedding. Let $N_{l, i}=\operatorname{ker}\left(j_{l}\right)_{* i}$, for $i=0,1$. As before, we obtain a unital separable $C^{*}$-algebra $B_{l+1} \supset B_{l}$ such that every pair of projections $p, q \in \bigcup_{k=1}^{\infty} M_{k}\left(B_{l}\right)$ with $[p]-[q] \in N_{l, 0}$ has the property that $[p]=[q]$ in $K_{0}\left(B_{l+1}\right)$, and every unitary $u \in B_{l}$ with $[u] \in N_{l, 1}$ has the property that $[u]=0$ in $K_{1}\left(B_{l+1}\right)$. Let $B_{0}$ be the closure of $\bigcup_{l=1}^{\infty} B_{l}$. Note that $B_{0}$ admits a full $\mathrm{O}_{2}$ embedding, say $j: B_{0} \rightarrow B$, and that $B_{0}$ is separable.

We claim that $j_{* i}$ is injective. Suppose that $p, q \in M_{k}\left(B_{0}\right)$ is a pair of projections for which $[p]-[q] \in \operatorname{ker} j_{* 0}$ and $[p]-[q] \neq 0$ in $B_{0}$. Without loss of generality, we may assume that $p, q \in M_{k}\left(B_{l}\right)$ for some large integer $l$. Then $[p]-[q]$ must be in the $\operatorname{ker}\left(j_{l}\right)_{* 0}$. By the construction, $[p]-[q]=0$ in $K_{0}\left(B_{l+1}\right)$. This would imply 
that $[p]-[q]=0$ in $K_{0}\left(B_{0}\right)$. Thus $j_{* 0}$ is injective. An exactly same argument shows that $j_{* 1}$ is also injective. The lemma follows.

Lemma 8.3. Let $B$ be a unital $C^{*}$-algebra which admits a full $\mathrm{O}_{2}$ embedding. Suppose that $G_{i} \subset K_{i}(B)$ and $F_{i}(k) \subset K_{i}(B, \mathbb{Z} / k \mathbb{Z})$ are countable subgroups such that the image of $F_{i}(k)$ in $K_{i-1}(B)$ is contained in $G_{i-1}$, for $i=0,1$ and $k=2,3, \ldots$ Then there exists a separable unital $C^{*}$-algebra $C \subset B$ admitting a full $\mathrm{O}_{2}$ embedding and such that $K_{i}(C) \supset G_{i}, K_{i}(C, \mathbb{Z} / k \mathbb{Z}) \supset F_{i}(k)$ and the embedding $j: C \rightarrow B$ induces an injective map

$$
j_{* i}: K_{i}(C) \rightarrow K_{i}(B)
$$

and an injective map

$$
j_{*}: K_{i}(C, \mathbb{Z} / k \mathbb{Z}) \rightarrow K_{i}(B, \mathbb{Z} / k \mathbb{Z}) \quad \text { for } k=2,3, \ldots
$$

Proof. By Lemma 8.2, there is a separable unital $C^{*}$-algebra $C_{1}$ admitting a full $\mathrm{O}_{2}$ embedding $j: C_{1} \rightarrow B$ and such that $K_{0}\left(C_{1}\right) \supset G_{0}, K_{1}\left(C_{1}\right) \supset G_{1}$ and $j$ induces an identity map on $K_{0}\left(C_{1}\right)$ and $K_{1}\left(C_{1}\right)$. Fix $k$, and let $\left\{x \in K_{i}\left(C_{1}\right): k x=0\right\}=$ $\left\{g_{1}^{(i)}, g_{2}^{(i)}, \ldots,\right\}$. Suppose that $\left\{s_{1}^{(i)}, s_{2}^{(i)}, \ldots,\right\}$ is a subset of $K_{i-1}(B, \mathbb{Z} / k \mathbb{Z})$ such that the map from $K_{i-1}(B, \mathbb{Z} / k \mathbb{Z})$ to $K_{i}(B)$ maps $s_{j}^{(i)}$ to $g_{j}^{(i)}$. For each $z^{(i)} \in$ $K_{i-1}\left(C_{1}, \mathbb{Z} / k \mathbb{Z}\right)$, there is $s_{j}^{(i)}$ such that

$$
z^{(i)}-s_{j}^{(i)} \in K_{i}(B) / k K_{i}(B) .
$$

Since $K_{i}\left(C_{1}\right)$ is countable, the set of all possible $z^{(i)}-s_{j}^{(i)}$ is countable. Thus one obtains a countable subgroup $G_{i}^{\prime}$ that contains $K_{i}\left(C_{1}\right)$ and for which $G_{i}^{\prime} / k K_{i}(B)$ contains the countable set just mentioned, as well as $F_{i}(k) \cap\left(K_{i}(B) / k K_{i}(B)\right)$ for each $k$. Since countably many countable sets is still countable, we obtain a countable subgroup $G_{i}^{(2)} \subset K_{i}(B)$ such that $G_{i}^{(2)}$ contains $G_{i}^{\prime}$ and $k K_{i}(B) \cap G_{i}^{(2)}=k G_{i}^{(2)}$, $k=1,2, \ldots$, and $i=0,1$. Note also $F_{i}(k) \cap\left(K_{i}(B) / k K_{i}(B)\right) \subset G_{i}^{(2)} / k K_{i}(B)$. By applying Lemma 8.2, we obtain a separable unital $C^{*}$-algebra $C_{2} \supset C_{1}$ such that $K_{i}\left(C_{2}\right) \supset G_{i}^{(2)}$ and an embedding from $C_{2}$ to $B$ gives an injective map on $K_{i}\left(C_{2}\right), i=0,1$. Repeating what we have done above, we obtain an increasing sequence of countable subgroups $G_{i}^{(n)} \subset K_{i}(B)$ such that $G_{i}^{(n)} \cap k K_{i}(B)=k G_{i}^{(n)}$ for all $k$ and $i=0,1$ and an increasing sequence of separable $C^{*}$-subalgebra s $C_{n}$ such that $K_{i}\left(C_{n}\right) \supset G_{i}^{(n)}$ and embeddings from $C_{n}$ into $B$ giving injective maps on $K_{i}\left(C_{n}\right), i=0,1$, and $n=1,2, \ldots$ Moreover $F_{i}^{(k)} \cap\left(K_{i}(B) / k K_{i}(B)\right) \subset$ $K_{i}\left(C_{n}\right) / k K_{i}(B)$. Let $C$ denote the closure of $\bigcup_{n} C_{n}$ and $j: C \rightarrow B$ be the embedding. Then $C$ is a separable unital $C^{*}$-algebra and $j_{* i}$ is an injective map, $i=0,1$. Since $C \supset C_{1}$ and $C_{1}$ is unital, $C$ admits a full $\mathrm{O}_{2}$ embedding. We claim that $K_{i}(C) \cap k K_{i}(B)=k K_{i}(C), k=1,2, \ldots$, and $i=0,1$. Note that $K_{i}(C)=\bigcup_{n} G_{i}^{(n)}$. Since $G_{i}^{(n)} \cap k K_{i}(B)=k G_{i}^{(n)} \subset k K_{i}(C)$, we see that $K_{i}(C) \cap k K_{i}(B)=k K_{i}(C)$, 
$i=0,1$. Thus $K_{i}(C) / k K_{i}(C)=K_{i}(C) / k K_{i}(B)$. Since

$$
K_{i}(C) / k K_{i}(B) \supset F_{i}^{(k)} \cap\left(K_{i}(B) / k K_{0}(B)\right),
$$

we conclude also that $K_{i}(C, \mathbb{Z} / k \mathbb{Z})$ contains $F_{i}(k)$. Since $j_{* i}$ is injective, $j$ induces an injective map from $K_{i}(C) / k K_{i}(C)$ into $K_{i}(B) / k K_{i}(B)$ for all integers $k \geq 1$. Using this and the fact that $j_{* i}: K_{i}(C) \rightarrow K_{i}(B)$ is injective, by chasing around the commutative diagram

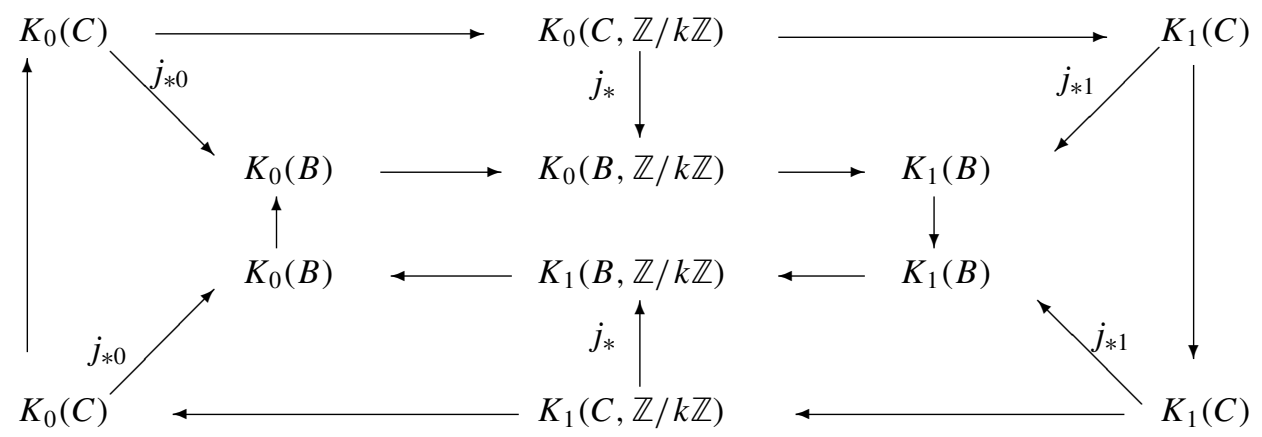

one sees that $j$ induces an injective map from $K_{i}(C, \mathbb{Z} / k \mathbb{Z})$ to $K_{i}(B, \mathbb{Z} / k \mathbb{Z})$.

Corollary 8.4. Without assuming that $B$ has a full $\mathrm{O}_{2}$ embedding, both Lemma 8.3 and Lemma 8.2 hold if we do not require that $C$ (or $B_{0}$ ) has a full $\mathrm{O}_{2}$ embedding.

Proof of Theorem 2.13. By Theorem 2.5, it suffices to show that, for each $x \in$ $K L(A, M(B) / B)$, there is a full monomorphism $h: A \rightarrow M(B) / B$ such that $[h]=x$. Put $Q=M(B) / B$. Since $A$ satisfies the AUCT, we may view $x$ as an element in $\operatorname{Hom}_{\Lambda}(\underline{K}(A), \underline{K}(Q))$. Note that $K_{i}(A)$ is a countable abelian group $(i=0,1)$. Let $G_{0}^{(i)}=\gamma(x)\left(K_{i}(A)\right), i=0,1$, where $\gamma: \operatorname{Hom}_{\Lambda}(\underline{K}(A), \underline{K}(Q)) \rightarrow$ $\operatorname{Hom}\left(K_{*}(A), K_{*}(Q)\right)$ is the surjective map. Then $G_{0}^{(i)}$ is a countable subgroup of $K_{i}(Q), i=0,1$. Consider the commutative diagram

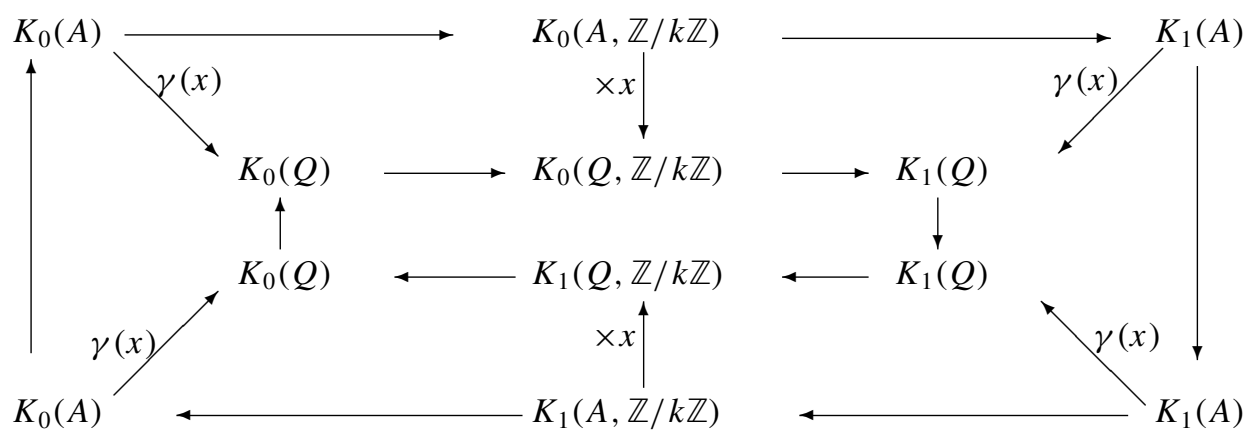

It follows from Lemma 8.3 that there is a unital $C^{*}$-algebra $C \subset Q$ which has a full $\mathrm{O}_{2}$ embedding such that $K_{i}(C) \subset G_{0}^{(i)}, K_{i}(C) \cap k K_{i}(Q)=k K_{i}(C), k=1,2, \ldots$, and $i=0,1$, and the embedding $j: C \rightarrow Q$ induces injective maps on $K_{i}(C)$ 
as well as on $K_{i}(C, \mathbb{Z} / k \mathbb{Z})$ for all $k$ and $i=0,1$. Moreover $K_{i}(C, \mathbb{Z} / k \mathbb{Z}) \supset$ $(\times x)\left(K_{i}(A, \mathbb{Z} / k \mathbb{Z})\right)$ for $k=1,2, \ldots$ and $i=0,1$. We have the commutative diagram

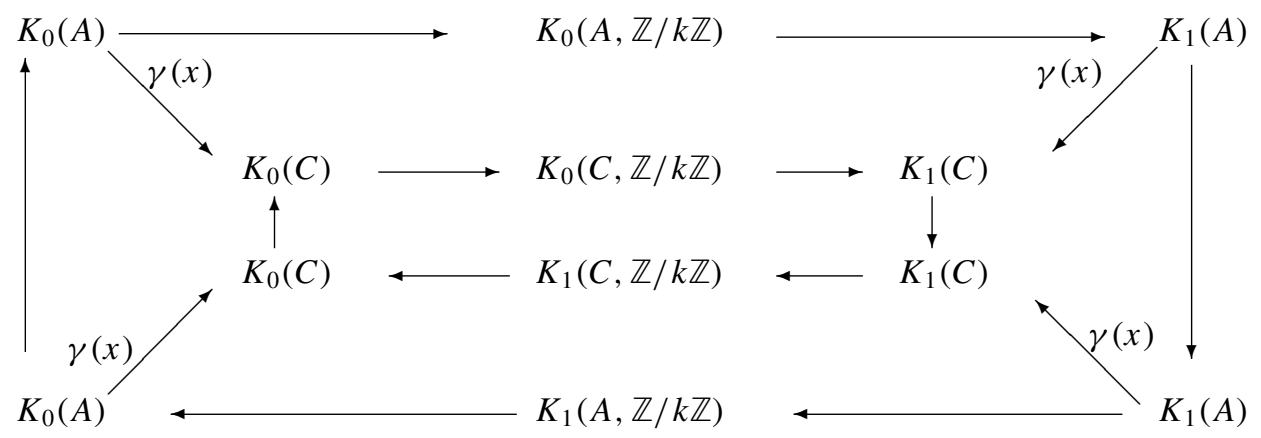

We will add two more maps on the above diagram. From the fact that the image of $K_{i}(A, \mathbb{Z} / k \mathbb{Z})$ under $\times x$ is contained in $K_{i}(C, \mathbb{Z} / k \mathbb{Z}),(k=2,3, \ldots, i=0,1)$, we obtain two maps $\beta_{i}: K_{i}(A, \mathbb{Z} / k \mathbb{Z}) \rightarrow K_{i}(C, \mathbb{Z} / k \mathbb{Z}), k=2,3, \ldots, i=0,1$ such that $j_{*} \circ \beta_{i}=\times x$ and obtain the commutative diagram

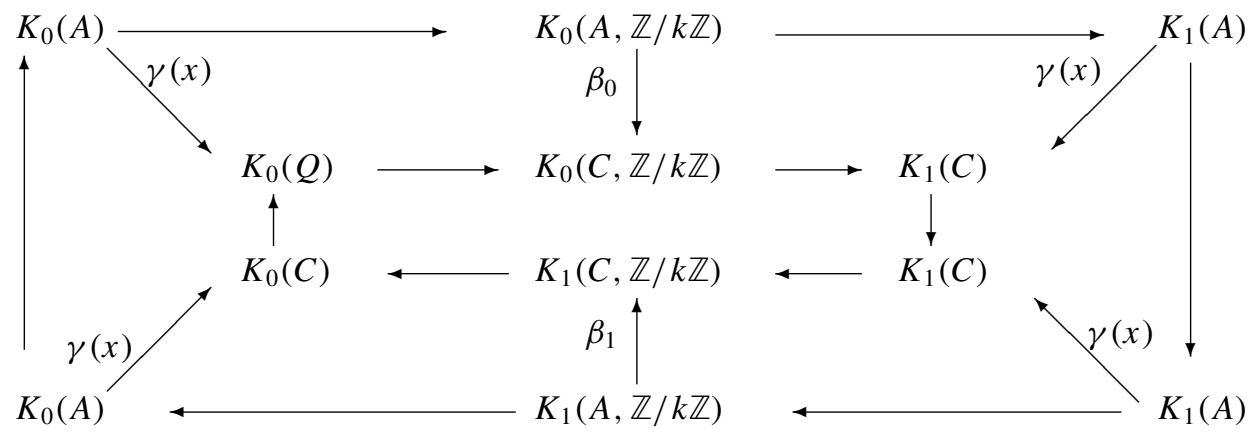

Consider the commutative diagram

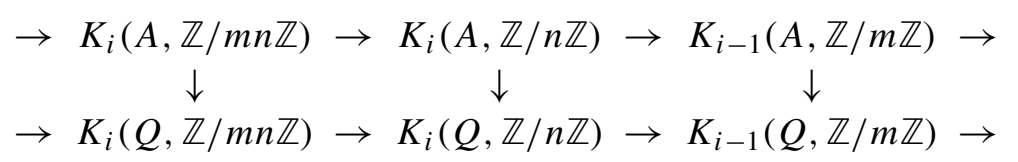

Since $j_{*} \circ \beta_{i}=\times x$ and all vertical maps in the diagram

$$
\begin{aligned}
& \rightarrow K_{i}(C, \mathbb{Z} / m n \mathbb{Z}) \rightarrow K_{i}(C, \mathbb{Z} / n \mathbb{Z}) \rightarrow K_{i-1}(C, \mathbb{Z} / m \mathbb{Z}) \rightarrow \\
& \rightarrow \begin{array}{c}
\downarrow \\
\downarrow \\
\downarrow
\end{array}(Q, \mathbb{Z} / m n \mathbb{Z}) \rightarrow K_{i}(Q, \mathbb{Z} / n \mathbb{Z}) \rightarrow K_{i-1}(Q, \mathbb{Z} / m \mathbb{Z}) \rightarrow
\end{aligned}
$$

are injective, we obtain the commutative diagram

$$
\begin{aligned}
& \rightarrow K_{i}(A, \mathbb{Z} / m n \mathbb{Z}) \rightarrow K_{i}(A, \mathbb{Z} / n \mathbb{Z}) \rightarrow K_{i-1}(A, \mathbb{Z} / m \mathbb{Z}) \rightarrow \\
& \rightarrow K_{i}(C, \mathbb{Z} / m n \mathbb{Z}) \rightarrow K_{i}(C, \mathbb{Z} / n \mathbb{Z}) \rightarrow K_{i-1}(C, \mathbb{Z} / m \mathbb{Z}) \rightarrow
\end{aligned}
$$


Thus we obtain $y \in K L(A, C)$ such that $y \times[j]=x$. Since $A$ satisfies the AUCT, one checks that $K L(A, C)=K L\left(A \otimes \mathrm{O}_{\infty}, C\right)$. This also follows from the fact that the unital embedding from $A \rightarrow A \otimes \mathrm{O}_{\infty}$ gives a $K K$-equivalence; see [Pimsner 1997]. It follows from [Lin 2007, 6.6, 6.7] that there exists a homomorphism $\phi: A \otimes \mathcal{O}_{\infty} \rightarrow C \otimes \mathscr{K}$ such that $[\phi]=y$. Define $\psi=\left.\phi\right|_{A \otimes 1}$. By the same result of Pimsner, one obtains that $[\psi]=y$. Since $A$ is unital, we may assume that the image of $\psi$ is in $M_{m}(C)$ for some integer $m \geq 1$. Since $C$ admits a full $\mathrm{O}_{2}$ embedding, $C$ has property $\mathrm{P} 2$. Thus $1_{m}$ is equivalent to a projection in $C$. Thus we may further assume that $\psi$ maps $A$ into $C$. Put $h_{1}=j \circ \psi$. To obtain a full monomorphism, we use the embedding $\iota: A \rightarrow \mathrm{O}_{2}$ given by [Kirchberg and Phillips 2000, Theorem 2.8]. Since $M(B) / B$ has property $\mathrm{P} 2$, we obtain a full monomorphism $\psi: \mathrm{O}_{2} \rightarrow$ $M(B) / B$. Let $e=\psi\left(1_{\mathbb{O}_{2}}\right)$. There is a partial isometry $w \in M_{2}(M(B) / B)$ such that $w^{*} w=1_{M(B) / B}$ and $w w^{*}=1 \oplus e$. Define $h=w^{*}\left(h_{1} \oplus \psi \circ \iota\right) w$. One checks that $[h]=\left[h_{1}\right]=x$ and $h$ is a full monomorphism.

Corollary 8.5. Let $A$ be a unital separable amenable $C^{*}$-algebra satisfying the $A U C T$. Let $B$ be a unital $C^{*}$-algebra which has property $P 2$. Then, for each $x \in$ $K L(A, B)$, there is a full monomorphism $h: A \rightarrow B$ such that $[h]=x$.

Proof. In the proof above, we may replace $M(B) / B$ by $B$.

Proof of Theorem 2.14. For the first part of the theorem, it suffices to show that every essential and full extension is absorbing. Let $\tau$ be a such extension. Following Elliott and Kucerovsky, we will show that $\tau$ is purely large. Denote $E=\tau^{-1}(A)$. Choose $c \in E \backslash C$. Then, by Lemma 3.3, $c$ is a full element. Since $M(C)$ has property $\mathrm{P} 1$, there exists $x \in M(B)$ such that $x^{*} c c^{*} x=1$. Therefore there exists a projection $p \leq c c^{*}$ for which there is $v \in M(B)$ such that $v^{*} v=1$ and $v v^{*}=p$. Note $\overline{c B c^{*}}=\overline{c M(B) c^{*}} \cap B$. Thus $p B p \subset \overline{c B c^{*}}$. Now $v^{*} p B p v=B$, so $p B p$ is stable and $p B p$ is full. Thus $\tau$ is purely large, hence absorbing. The last part of the theorem follows from the next corollary.

Corollary 8.6. Let $A$ be a separable unital amenable $C^{*}$-algebra and $C$ a unital $C^{*}$-algebra, and set $B=C \otimes \mathcal{K}$. Then $\operatorname{Ext}(A, B)$ is the same set as unitary equivalence classes of essential and full extensions of $A$ by $B$.

Proof. It suffices to show that given any element $x \in \operatorname{Ext}(A, B)$, there exists an essential and full extension $\tau: A \rightarrow M(B) / B$ so that $[\tau]=x$. There exists an essential extension $\tau_{1}: A \rightarrow M(B) / B$ such that $\left[\tau_{1}\right]=x$. Take a monomorphism $j: A \rightarrow \mathrm{O}_{2}$ (see [Kirchberg and Phillips 2000]). Let $h: \mathrm{O}_{2} \rightarrow M(\mathscr{K})$ be a monomorphism (given by a faithful representation of $\mathrm{O}_{2}$ on a separable Hilbert space). Let $\phi: M(\mathscr{K}) \rightarrow M(B)$ be the standard unital embedding and $\pi: M(B) \rightarrow M(B) / B$ be the quotient map. Then $\tau_{2}=\pi \circ \phi \circ h \circ j$ gives a full essential trivial extension. It follows that $\tau=\tau_{1} \oplus \tau_{2}$ is an full essential extension. Since $\left[\tau_{2}\right]=0,[\tau]=\left[\tau_{1}\right]=x$. 
Remark 8.7. Let $B$ be a nonstable, nonunital but $\sigma$-unital $C^{*}$-algebra. Suppose that $M(B) / B$ has properties $\mathrm{P} 1, \mathrm{P} 2$ and $\mathrm{P} 3$, and suppose that $\tau: A \rightarrow M(B) / B$ is an essential and full extension. One should not expect that such extension is purely large in general. Let $0 \rightarrow B \rightarrow E \rightarrow A \rightarrow 0$ be an essential and full extension corresponding to $\tau$. Recall that the extension is purely large if $c B c^{*}$ contains a $C^{*}$-subalgebra which is stable and $c B c^{*}$ is full in $B$; see [Elliott and Kucerovsky 2001]. Given any element $c \in E \backslash B, \pi(c)$ is full in $M(B) / B$. But, in general, $c$ need not be full in $M(B)$, nor does $c B c^{*}$ need to be full in $B$. Examples are easily seen in the case that $B=c_{0}(C)$, where $C$ is a unital purely infinite simple $C^{*}$-algebra. Suppose that $0 \rightarrow c_{0}(C) \rightarrow E \rightarrow A \rightarrow 0$ is a full extension and $c^{\prime} \in E \backslash c_{0}(C)$. Write $c^{\prime}=\left\{c_{n}^{\prime}\right\} \in l^{\infty}(C)$. Define $c_{n}=c_{n}^{\prime}$ if $n \geq N>1$ and $c_{n}=0$ if $n \leq N$. Put $c=\left\{c_{n}\right\}$. Then $c \in E \backslash c_{0}(C)$. However, it is clear that $c c_{0}(C) c^{*}$ is not full in $c_{0}(C)$. By Theorem 7.5, the full extension $\tau$ is approximately absorbing in the sense of Theorem 7.5 but not purely large. It should be also noted that, even if $c^{*} B c$ is full for all $c \in E \backslash B$, the full extension may not be purely large. Let $B$ be a nonstable, nonunital but $\sigma$-unital simple $C^{*}$-algebra with continuous scale (see [Lin 2004b] for more examples). Then $B$ may be stably finite. No hereditary $C^{*}$-subalgebra of $B$ contains a stable $C^{*}$-subalgebra. So none of the essential extensions of a unital separable amenable $C^{*}$-algebra $A$ by $B$ could be possibly purely large in the sense of [Elliott and Kucerovsky 2001]; nevertheless, all of these extensions are approximately absorbing in the sense of Theorem 7.5 (and many of them are actually absorbing: for example, when $A=C(X)$ ).

\section{Acknowledgement}

This work started in summer 2003 when the author was visiting East China Normal University. It is partially supported by the U.S. National Science Foundation and Shanghai Academic Priority Disciplines.

\section{References}

[Akemann et al. 1986] C. A. Akemann, J. Anderson, and G. K. Pedersen, "Excising states of $C^{*}$ algebras”, Canad. J. Math. 38:5 (1986), 1239-1260. MR 88d:46107 Zbl 0704.46043

[Blackadar 1998] B. Blackadar, K-theory for operator algebras, 2nd ed., Math. Sci. Res. Inst. Publ. 5, Cambridge University Press, Cambridge, 1998. MR 99g:46104 Zbl 0913.46054

[Blackadar et al. 1991] B. Blackadar, M. Dădărlat, and M. Rørdam, “The real rank of inductive limit $C^{*}$-algebras”, Math. Scand. 69:2 (1991), 211-216. MR 93e:46067 Zbl 0776.46025

[Brown 1984] L. G. Brown, "The universal coefficient theorem for Ext and quasidiagonality", pp. 60-64 in Operator algebras and group representations (Neptun, 1980), vol. 1, edited by G. Arsene et al., Monogr. Stud. Math. 17, Pitman, Boston, 1984. MR 85m:46066 Zbl 0548.46055

[Brown and Elliott 1982] L. G. Brown and G. A. Elliott, "Extensions of AF-algebras are determined by $K_{0}$ ”, C. R. Math. Rep. Acad. Sci. Canada 4:1 (1982), 15-19. MR 83c:46059 Zbl 0474.46060 
[Brown et al. 1973a] L. G. Brown, R. G. Douglas, and P. A. Fillmore, "Extensions of $C^{*}$-algebras, operators with compact self-commutators, and K-homology”, Bull. Amer. Math. Soc. 79 (1973), 973-978. MR 49 \#11265 Zbl 0277.46052

[Brown et al. 1973b] L. G. Brown, R. G. Douglas, and P. A. Fillmore, "Unitary equivalence modulo the compact operators and extensions of $C^{*}$-algebras", pp. 58-128 in Proceedings of a Conference on Operator Theory (Halifax, N.S., 1973), edited by P. A. Fillmore, Lecture Notes in Math. 345, Springer, Berlin, 1973. MR 52 \#1378 Zbl 0277.46053

[Dadarlat and Loring 1996] M. Dadarlat and T. A. Loring, "A universal multicoefficient theorem for the Kasparov groups”, Duke Math. J. 84:2 (1996), 355-377. MR 97f:46109 Zbl 0881.46048

[Effros 1981] E. G. Effros, Dimensions and $C^{*}$-algebras, CBMS Regional Conference Series in Math. 46, Conference Board of the Mathematical Sciences, Washington, DC, 1981. MR 84k:46042 Zbl 0475.46050

[Elliott and Handelman 1989] G. A. Elliott and D. E. Handelman, "Addition of $C^{*}$-algebra extensions”, Pacific J. Math. 137:1 (1989), 87-121. MR 90e:46047 Zbl 0685.46042

[Elliott and Kucerovsky 2001] G. A. Elliott and D. Kucerovsky, "An abstract Voiculescu-BrownDouglas-Fillmore absorption theorem”, Pacific J. Math. 198:2 (2001), 385-409. MR 2002i:46052 Zbl 1058.46041

[Goodearl and Handelman 1982] K. R. Goodearl and D. E. Handelman, "Stenosis in dimension groups and AF $C^{*}$-algebras", J. Reine Angew. Math. 332 (1982), 1-98. MR 83m:46101 Zbl 0489. 46052

[Husemoller 1966] D. Husemoller, Fibre bundles, McGraw-Hill, New York, 1966. MR 37 \#4821 Zbl 0144.44804

[Kirchberg 1996] E. Kirchberg, "Classification of purely infinite simple $C^{*}$-algebras by Kasparov groups", preprint, third draft, 1996.

[Kirchberg and Phillips 2000] E. Kirchberg and N. C. Phillips, "Embedding of exact $C^{*}$-algebras in the Cuntz algebra $0_{2}$ ”, J. Reine Angew. Math. 525 (2000), 17-53. MR 2001d:46086a Zbl 0973. 46048

[Kirchberg and Rørdam 2000] E. Kirchberg and M. Rørdam, "Non-simple purely infinite $C^{*}$-algebras", Amer. J. Math. 122:3 (2000), 637-666. MR 2001k:46088 Zbl 0968.46042

[Lin 1995a] H. X. Lin, $C^{*}$-algebra extensions of $C(X)$, Mem. Amer. Math. Soc. 550, Amer. Math. Soc., Providence, RI, 1995. MR 96b:46098 Zbl 0859.46038

[Lin 1995b] H. X. Lin, "Extensions by $C^{*}$-algebras of real rank zero. II”, Proc. London Math. Soc. (3) 71:3 (1995), 641-674. MR 96j:46072 Zbl 0837.46042

[Lin 1997] H. Lin, "Extensions of $C(X)$ by simple $C^{*}$-algebras of real rank zero", Amer. J. Math. 119:6 (1997), 1263-1289. MR 98m:46088 Zbl 0895.46034

[Lin 1998] H. Lin, "Extensions by $C^{*}$-algebras of real rank zero. III", Proc. London Math. Soc. (3) 76:3 (1998), 634-666. MR 99j:46071 Zbl 0911.46043

[Lin 2001] H. Lin, An introduction to the classification of amenable $C^{*}$-algebras, World Scientific, River Edge, NJ, 2001. MR 2002k:46141 Zbl 1013.46055

[Lin 2004a] H. Lin, "A separable Brown-Douglas-Fillmore theorem and weak stability", Trans. Amer. Math. Soc. 356:7 (2004), 2889-2925. MR 2005d:46116 Zbl 1053.46032

[Lin 2004b] H. Lin, "Simple corona $C^{*}$-algebras", Proc. Amer. Math. Soc. 132:11 (2004), 32153224. MR 2005e:46100 Zbl 1049.46040

[Lin 2005a] H. Lin, "An approximate universal coefficient theorem”, Trans. Amer. Math. Soc. 357:8 (2005), 3375-3405. MR 2006a:46068 Zbl 1068.46039 
[Lin 2005b] H. Lin, "Extensions by simple $C^{*}$-algebras: quasidiagonal extensions", Canad. J. Math. 57:2 (2005), 351-399. MR 2005m:46094 Zbl 1087.46039

[Lin 2007] H. Lin, "Semiprojectivity in purely infinite simple $C^{*}$-algebras", Canad. J. Math. (2007). To appear.

[Pedersen 1979] G. K. Pedersen, $C^{*}$-algebras and their automorphism groups, London Math. Soc. Monogr. 14, Academic Press, London, 1979. MR 81e:46037 Zbl 0416.46043

[Pimsner 1997] M. V. Pimsner, "A class of $C^{*}$-algebras generalizing both Cuntz-Krieger algebras and crossed products by $\mathbb{Z}$ ", pp. 189-212 in Free probability theory (Waterloo, ON, 1995), edited by F. probability theory, Fields Inst. Commun. 12, Amer. Math. Soc., Providence, RI, 1997. MR 97k:46069 Zbl 0871.46028

[Pimsner et al. 1979] M. Pimsner, S. Popa, and D. Voiculescu, "Homogeneous $C^{*}$-extensions of $C(X) \otimes K(H)$, I", J. Operator Theory 1:1 (1979), 55-108. MR 82e:46093a Zbl 0455.46060

[Pimsner et al. 1980] M. Pimsner, S. Popa, and D. Voiculescu, "Homogeneous $C^{*}$-extensions of $C(X) \otimes K(H)$, II”, J. Operator Theory 4:2 (1980), 211-249. MR 82e:46093b Zbl 0484.46059

[Rørdam 1994] M. Rørdam, "A short proof of Elliott's theorem: $\mathrm{O}_{2} \otimes \mathrm{O}_{2} \cong \mathrm{O}_{2}$ ", C. R. Math. Rep. Acad. Sci. Canada 16:1 (1994), 31-36. MR 95d:46064 Zbl 0817.46061

[Rørdam 2002] M. Rørdam, "Classification of nuclear, simple $C^{*}$-algebras", pp. 1-145 in Classification of nuclear $C^{*}$-algebras. Entropy in operator algebras, Encyclopaedia Math. Sci. 126, Springer, Berlin, 2002. MR 2003i:46060 Zbl 0826.46064

[Rosenberg and Schochet 1987] J. Rosenberg and C. Schochet, "The Künneth theorem and the universal coefficient theorem for Kasparov's generalized $K$-functor", Duke Math. J. 55:2 (1987), 431-474. MR 88i:46091 Zbl 0644.46051

[Schochet 1984] C. Schochet, “Topological methods for $C^{*}$-algebras, IV: Mod $p$ homology", $P a$ cific J. Math. 114:2 (1984), 447-468. MR 86g:46103 Zbl 0491.46062

[Zhang 1992] S. Zhang, "Certain $C^{*}$-algebras with real rank zero and their corona and multiplier algebras, I”, Pacific J. Math. 155:1 (1992), 169-197. MR 94i:46093

Received April 16, 2004.

HUAXIN LIN

DEPARTMENT OF MATHEMATICS

EAST CHINA NORMAL UNIVERSITY

SHANGHAI

CHINA

Deceased January 14, 2007

hlin@uoregon.edu 